\title{
REVIEW ARTICLE OPEN Deformation imaging and rotational mechanics in neonates: a guide to image acquisition, measurement, interpretation, and reference values
}

\author{
Afif El-Khuffash ${ }^{1,2}$, Ulf Schubert ${ }^{3}$, Philip T. Levy ${ }^{4,5}$, Eirik Nestaas ${ }^{6,7,8}$ and Willem P. de Boode ${ }^{9}$ on behalf of the European Special Interest \\ Group 'Neonatologist Performed Echocardiography' (NPE)
}

\begin{abstract}
Advances in neonatal cardiac imaging permit a more comprehensive assessment of myocardial performance in neonates that could not be previously obtained with conventional imaging. Myocardial deformation analysis is an emerging quantitative echocardiographic technique to characterize global and regional ventricular function in neonates. Cardiac strain is a measure of tissue deformation and strain rate is the rate at which deformation occurs. These measurements are obtained in neonates using tissue Doppler imaging (TDI) or two-dimensional speckle tracking echocardiography (STE). There is an expanding body of literature describing longitudinal reference ranges and maturational patterns of strain values in term and preterm infants. A thorough understanding of deformation principles, the technical aspects, and clinical applicability is a prerequisite for its routine clinical use in neonates. This review explains the fundamental concepts of deformation imaging in the term and preterm population, describes in a comparative manner the two major deformation imaging methods, provides a practical guide to the acquisition and interpretation of data, and discusses their recognized and developing clinical applications in neonates.
\end{abstract}

Pediatric Research (2018) 84:S30-S45; https://doi.org/10.1038/s41390-018-0080-2

\section{INTRODUCTION}

Characterization of myocardial adaptation with echocardiography during the critical periods of development in neonates is important for recognition and management of circulatory disturbances. ${ }^{1}$ Conventional measures of left ventricular (LV) function, such as shortening (SF) and ejection fraction (EF), assess the changes in cavity dimensions, but are insufficient to detect overt dysfunction in a timely manner, because their measurements are influenced by image quality and inadequate reproducibility and standardization in neonates. ${ }^{2}$ Similarly, conventional techniques to estimate right ventricular (RV) performance rely largely on quantitative estimates and qualitative predictions that are limited because of the unique three-dimensional structure of the chamber., Although conventional echocardiography with tissue Doppler (TD) velocity is considered to be reliable for ventricular wall motion analysis, the visual estimation of wall motion is very subjective and cannot be utilized as an aid to clinical assessment. ${ }^{5}$ Many challenges also exist for serially monitoring the cardiovascular status of preterm infants and sick term infants, largely due to the relative insensitivity of clinical markers (such as blood pressure and capillary refill time) in defining hemodynamic compromise. ${ }^{6,7}$ For the management of a broad range of heart diseases, a quantitative, reproducible approach for the serial assessment of cardiac function is of paramount importance in neonates. ${ }^{8}$

The concept of deformation imaging is a novel technique, recently introduced to the field of neonatology, and can be measured by either speckle tracking echocardiography (STE) or tissue Doppler imaging (TDI). ${ }^{9-11}$ Myocardial strain $(\varepsilon)$ is a measure of tissue deformation and strain rate (SR) is the rate at which deformation occurs. Both are feasible and reproducible markers of global and regional performance that provide fundamental information on myocardial properties and mechanics that would otherwise be unavailable with conventional imaging. ${ }^{3,9-12}$ Myocardial strain can be measured in terms of three normal strains (longitudinal, radial, and circumferential) and six shear strains. ${ }^{13}$ Currently, only normal strain and shear strain in the circumferentiallongitudinal plane (rotational mechanics) have been investigated for clinical use in neonates. ${ }^{9}$ A thorough understanding of the basic principles of deformation imaging, recognition of their applicability in term and preterm infants, strengths, and limitations is essential for advancing those techniques to routine clinical care. This review explains the fundamental concepts of deformation imaging, describes in a comparative manner the two major

\footnotetext{
${ }^{1}$ Department of Neonatology, The Rotunda Hospital, Dublin, Ireland; ${ }^{2}$ Department of Pediatrics, The Royal College of Surgeons in Ireland, Dublin, Ireland; ${ }^{3}$ Department of Clinical Science, Intervention and Technology, Karolinska Institutet, Stockholm, Sweden; ${ }^{4}$ Department of Pediatrics, Washington University School of Medicine, Saint Louis, MO, USA; ${ }^{5}$ Department of Pediatrics, Goryeb Children's Hospital, Morristown, NJ, USA; ${ }^{6}$ Institute of Clinical Medicine, Faculty of Medicine, University of Oslo, Oslo, Norway; ${ }^{7}$ Department of Cardiology and Center for Cardiological Innovation, Oslo University Hospital, Rikshospitalet, Oslo, Norway; ${ }^{8}$ Department of Paediatrics, Vestfold Hospital Trust, Tønsberg, Norway and ${ }^{9}$ Department of Neonatology, Radboud University Medical Center, Radboud Institute for Health Sciences, Amalia Children's Hospital, Nijmegen, The Netherlands Correspondence: Willem P. de Boode (willem.deboode@radboudumc.nl)

Members of the European Special Interest Group 'Neonatologist Performed Echocardiography' (NPE), endorsed by the European Society for Paediatric Research (ESPR) and
} European Board of Neonatology (EBN) are listed in the Appendix. 
deformation imaging methods, provides a practical guide to the acquisition and interpretation of data, and discusses the clinical applications and available reference ranges in the term and preterm population.

\section{BASIC CONCEPTS OF MYOCARDIAL DEFORMATION}

Deformation refers to the change in the shape of the myocardium from its baseline shape at end-diastole to its deformed shape at end-systole. ${ }^{9}$ This occurs in response to sarcomere shortening due to contraction (Fig. 1). The deformation leads to a reduction in cavity size and ejection of blood from the ventricle. Myocardial strain $(\varepsilon)$ is a dimensionless index that is defined as the relative tissue deformation under an applied force, and is expressed as a percentage (\%; see Geyer et al. $\left.{ }^{14}\right)$. SR $\left(\mathrm{s}^{-1}\right)$ is the first derivative of strain, or the speed at which deformation occurs in systole. ${ }^{14}$ When applied to the neonatal myocardium, strain and SR are used as measures of global and regional ventricular function (Figs. 2 and 9).

Myocardial strain can be measured in terms of "normal" strain and "shear" strain. ${ }^{13}$ Normal strain is caused by forces that act perpendicular to the surface of the myocardial wall, resulting in stretching or contraction without skewing of the volume. ${ }^{15}$ There are three types of normal strain; longitudinal, radial, and circumferential. Conversely, forces causing shear strain act parallel to the surface of the wall and lead to a shift of volume borders relative to one another as delineated by a "shear" angle. ${ }^{15}$ There are six forms of shear strain grouped into three categories; circumferential-longitudinal, circumferential-radial, and longitudinal-radial. Myocardial shear in the circumferentiallongitudinal plane results in twist or torsional deformation of the LV during ejection. Only the three normal forms of strain and circumferential-longitudinal shear strain (rotational mechanics) have been investigated for clinical use in neonates. ${ }^{9}$

\section{METHODS OF DEFORMATION ASSESSMENT}

LV and RV deformation patterns differ based on their own unique myoarchitectural fiber orientation. The LV myocardium consists of circumferential fibers in the midwall layer and longitudinal fibers
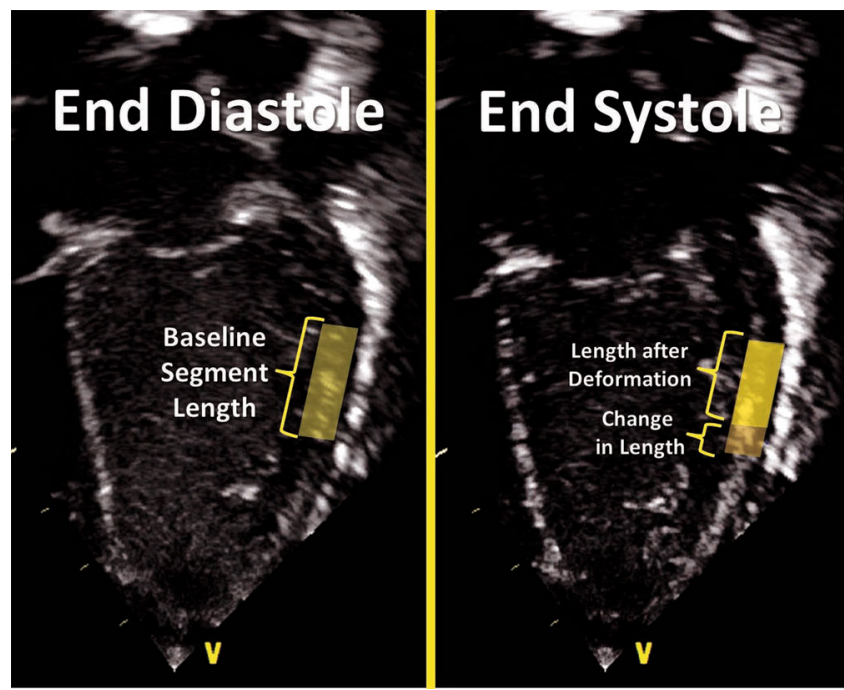

Fig. 1 Principles of deformation. Longitudinal strain refers to the change in length of a segment from its baseline length in enddiastole to its deformed shape in systole. Strain refers to the degree of change in shape relative to the baseline and is expressed in $\%$. Shortening reflects negative values and lengthening positive values. In this image, shortening of the mid-segment of the LV free wall is illustrated in the endocardial and epicardial layers. ${ }^{16}$ During myocardial contraction, the LV wall shortens and thickens with LV deformation occurring in three directions: (i) longitudinal shortening that is directed from base to apex in the apical four-chamber view, (ii) circumferential shortening along the circular perimeter observed in a parasternal short-axis view, and (iii) radial thickening directed toward the center of the LV cavity measured in the parasternal short axis $\left({ }^{14}\right.$; Fig. 3). Deformation is assigned a negative sign for shortening (in longitudinal and circumferential planes) and a positive sign for thickening in the radial plane.

In the circumferential-longitudinal plane, the net difference in the systolic rotation of the myocardium between the apical and basal short-axis plane is referred to as twist (measured in degrees) and represents the wringing motion of the LV during systole. If normalized to the distance between the respective image planes,

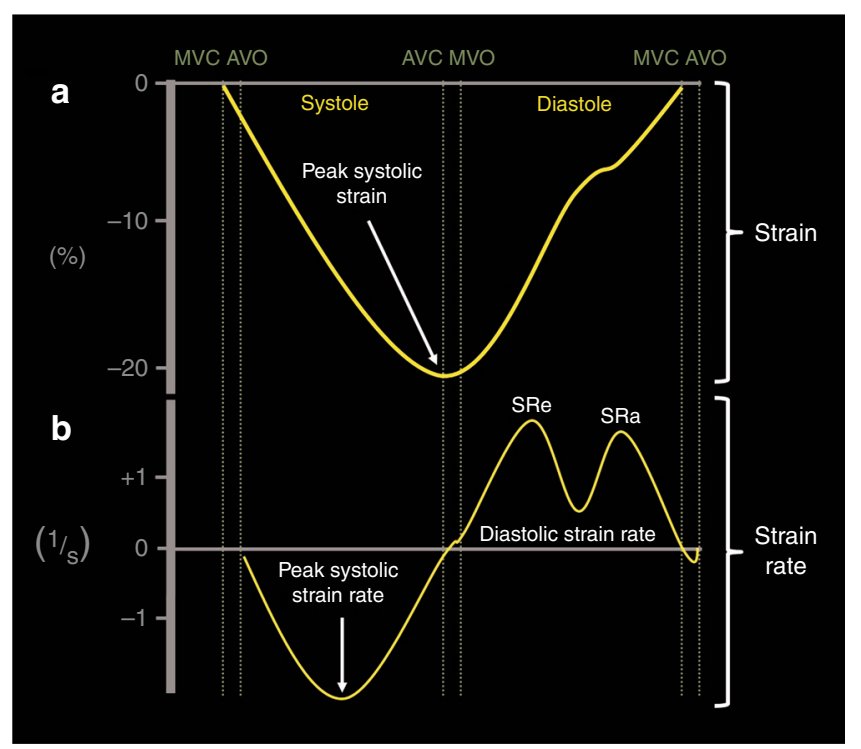

Fig. 2 Strain and strain curves in one cardiac cycle in the ventricle. a Strain usually peaks during end-systole at aortic valve closure (AVC) and returns to baseline during diastole at mitral valve closure (MVC). b Strain rate usually peaks in mid-systole and returns to baseline at AVC when no deformation occurs. During diastole, the rate of strain returning to baseline is biphasic. MVO mitral valve opening, AVO aortic valve closure, SRe early diastolic strain rate, SRa late diastolic strain rate (during atrial contraction)

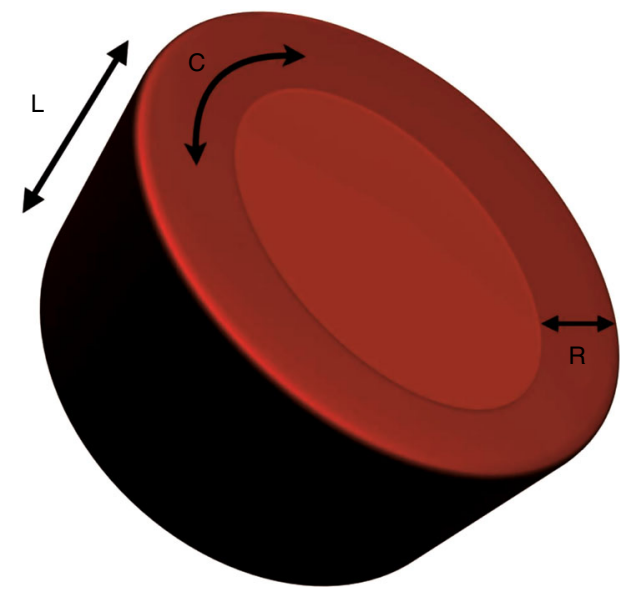

Fig. 3 LV deformation. LV deformation occurring in three directions; $\mathrm{L}$ longitudinal, C circumferential, $\mathrm{R}$ radial 
it is referred to as torsion $(\% / \mathrm{cm})$. LV rotational mechanics (twist and torsion) are assessed by STE.

Compared with the LV, the RV myofiber architecture is composed of superficial oblique and dominant deep longitudinal layers. The myofibers in the RV are aligned in a more longitudinal direction than in the $L V$, and as the dominant pattern of RV deformation, longitudinal shortening provides the major contribution to stroke volume during systole and is a more sensitive indicator of RV dysfunction. ${ }^{3,13,17}$ Deformation in the circumferential and radial directions in the RV may prove to be a valuable measure of function in certain neonatal conditions (i.e., congenital heart disease), but there is a paucity of studies that use these measures in clinical practice and those studies have not been able to demonstrate significant reliability in neonates. ${ }^{18,19}$

There are two established methods for assessing and calculating deformation entitled Lagrangian strain and Eulerian (natural) strain. ${ }^{14,20}$ Lagrangian strain refers to the change in length relative to an unstressed baseline length, against which all subsequent deformation will be measured. ${ }^{21}$ Since Lagrangian strain is measured as the separation distance between two regions of myocardium relative to the original separation distance in enddiastole, it is not affected by the heart rate (HR). ${ }^{22}$ STE lends itself more readily to the calculation of Lagrangian strain, since the baseline length is always known and can easily be used as a reference. $^{14}$ Eulerian strain calculation is based on a reference length that is different at each interrogation time point, i.e., each color TD frame, and is better suited for use with TDI. Natural and Lagrangian strains are related so that one can be converted into the other. STE software packages will report Lagrangian strain, but natural strain (i.e., TD) can be derived from STE by conversion from the Lagrangian strain. Studies that utilized strain and SR measures to characterize ventricular function in neonates must therefore indicate the software package and the type of strain or SR.

\section{INFLUENCES OF MYOCARDIAL CONTRACTILITY AND LOAD DEPENDENCE}

Deformation is affected by several factors that should be considered when using strain imaging in clinical practice. Specifically, global and regional strains (\%) are influenced by preload (which increases wall strain) and afterload (which reduces wall strain; ${ }^{14}$ ). Compared to strain, $S R$ is thought to be less dependent on loading conditions, and is a more accurate reflection of intrinsic myocardial contractile function. ${ }^{23}$ Preclinical studies in animal models have demonstrated that preload has a positive impact on strain, whereas increasing afterload is associated with its reduction. ${ }^{23-25}$ In preterm clinical studies, surgical ligation of patent ductus arteriosus (PDA) results in a sudden elevation in LV afterload and a reduction in LV preload that significantly decreases LV strain in the immediate postoperative period. $^{2}$ In the early transitional period in preterm infants, there is a negative correlation between strain and measures of afterload and positive correlation between strain and measures of preload. ${ }^{19,26}$ Antenatal magnesium sulfate administration is associated with lower systemic vascular resistance (SVR) and higher myocardial function as measured by strain imaging, but SR appeared to be less influenced by loading conditions, further validating the lower SR dependency on loading conditions ( ${ }^{27}$; Fig. 4).

\section{VARIABILITY AND STANDARDIZATION OF DEFORMATION IMAGING}

Three factors modulate variability in deformation imaging; these include variability in image acquisition, intra- and inter-observer variability in post-acquisition processing, and differences between echocardiographic equipment and proprietary software for image analysis. ${ }^{3,28,29}$ This review addresses the first two factors (proper image acquisition and validation of deformation measurements),
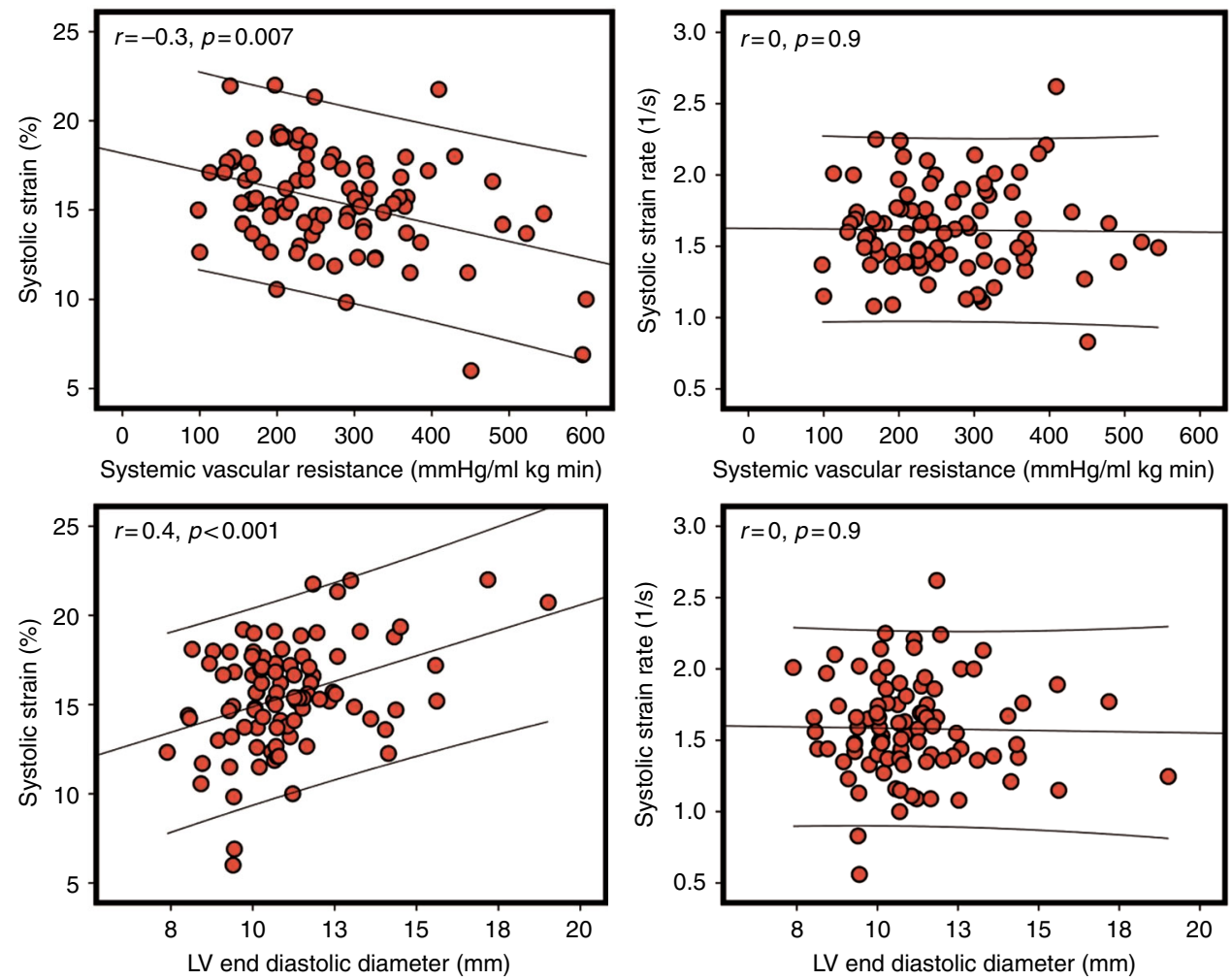

Fig. 4 The relationship between loading conditions and deformation parameters. There is a negative relationship between strain and systemic vascular resistance (a surrogate of afterload) but a positive relationship between strain and left ventricle end-diastolic diameter (a surrogate of preload). Note the lack of relationship between systolic strain rate and loading measures (data set from ref. ${ }^{26}$ ) 
but in light of the push to standardize the acquisition of these measures and reduce inter-vendor differences and ambiguities, ${ }^{21,30}$ it is important for the reader to review any details of hardware settings, manual settings, and local imaging protocols to get a better understanding of the values presented.

To measure and calculate myocardial strain and SR, there are two different types of echocardiographic imaging modalities: TDI and STE. First introduced as a post-processing feature of TDI with velocity data converted to strain and SR, strain imaging information has more recently also been derived from STE computer processing. ${ }^{31}$ These imaging modalities derive information on myocardial strain and SR from two fundamentally different ways and will be considered separately.

\section{TISSUE DOPPLER DEFORMATION IMAGING}

Principles and validation in neonates

Echocardiographic strain was first derived from TDI velocity data using the Doppler equation to convert ultrasound frequency shifts to velocity information along the scan lines. ${ }^{31}$ In the longitudinal plane of the ventricle, there is a velocity gradient from the base of the heart toward the apex. Basal myocardial tissue moves at a higher velocity (toward the apex in systole) than myocardial tissue at the apex due to the tethering effects and the stationary position of the apex (Fig. 5). TD-derived (longitudinal) deformation imaging calculates SR by assessing the difference in velocity (the velocity gradient) between points along the longitudinal plane. Strain is then assessed by integrating the SR values by time. Only velocities along (parallel to) the beam of the ultrasound are measured by the TD method; therefore, deformation indices measured using TD are highly dependent on the angle of insonation. Due to the high temporal resolution of this technique, TD is well suited for measurement of SR values in neonates (with a higher baseline HR) as it employs a calculation method that utilizes the high TD frame rates (FRs) (>180 frames per second; ${ }^{26,32}$ ). In neonates, TDI- derived deformation values can be obtained from several regions of the heart. Longitudinal deformation can be measured from most parts of the LV and RV and is more often measured in neonates. ${ }^{26,32}$ Circumferential and radial deformation can only be assessed in a few LV regions. ${ }^{33}$

To characterize ventricular function with longitudinal strain, some studies have reported values from many heart segments, ${ }^{33-38}$ while others have obtained values from the basal segment of the LV and RV free walls, in addition to the septum. ${ }^{26,39-41}$ The LV base remains the most challenging segment to assess with TD. The reliability of the results obtained from the LV base is reduced because artifacts arising from the lungs often obscure the base. This high dependency on the requirement for clear imaging of the myocardial walls and the angle dependency (it is difficult to maintain an angle insonation less than $20^{\circ}$ between the ultrasound beam and the LV wall), coupled with the artifacts of extra-cardiac structures and image dropouts, can lead to over- and underestimation of strain and SR values. ${ }^{42}$

The feasibility and reproducibility of TD-derived deformation parameters have been established in term and preterm neonates. ${ }^{11,36,37,41}$ The most reproducible measurements are assessed in longer segments and when the LV base free wall is avoided as site of measurement. ${ }^{35}$ Nestaas et al. conducted the first studies of reproducibility in term infants and revealed moderate reproducibility in obtaining strain and systolic SR with intraclass correlation coefficients (ICC) ranging between 0.6-0.7 for intra-observer and $0.4-0.5$ for inter-observer repeated measures. ${ }^{36,43}$ With enhanced image optimization techniques, reliability data have improved in term neonates for basal strain with coefficients of variation (COV) values $<15 \%$ and ICC values $>0.75 .^{35,38,40}$ In preterm infants, measurements are feasible in about $90 \%$ of studies when adequate imaging quality is achieved. ${ }^{41}$ Poon et al. reported COV values of $<5 \%$ for LV, septal and RV basal strain in preterm infants. ${ }^{38}$ Helfer et al. further illustrated more modest reproducibility results in preterm infants; the septum showed the best COV

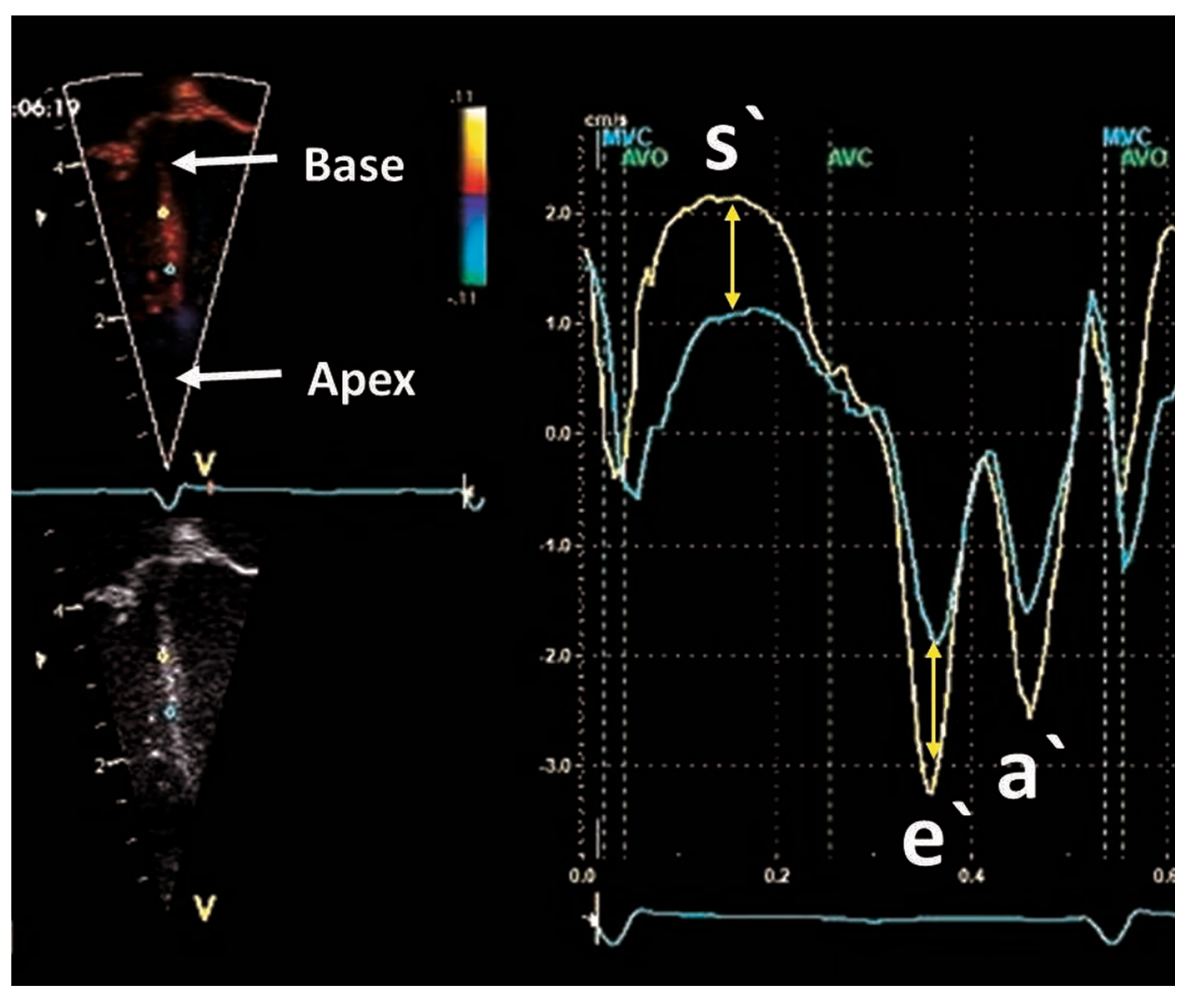

Fig. 5 Difference in velocity between two points along the long axis of the septum. The curves show tissue velocities by tissue Doppler during the cardiac cycle. The point closer to the base (yellow) has a higher systolic and diastolic velocity when compared with the point closer to the base (green). The difference in velocity is used to calculate strain rate and derive strain of that segment bordered by the two points 
ranging from $10 \%$ to $34 \%$ for intra- and $22 \%$ to $27 \%$ for interobserver variability, whereas the left wall had a higher and wider range of values $(17-57 \%$ for intra- and $29-60 \%$ for inter-observer variability; $\left.{ }^{39}\right)$. James et al. demonstrated that reproducibility of basal longitudinal strain and SR measurements in the RV and septum were more favorable than the LV in preterm infants. ${ }^{41}$ All of these studies in preterm and term infants consistently show reduced reproducibility of LV basal strain values, and cited poor image quality and difficulty in obtaining an angle on insonation $<20^{\circ}$ as the main reasons.

Image acquisition and offline data measurement

To obtain reliable deformation values, strain by TD imaging and post-processing analysis protocols have been developed and implemented in neonates. ${ }^{36,43}$ A clear electrocardiogram (ECG) signal with a well-defined QRS complex is necessary for obtaining a complete cardiac cycle for offline processing. Pulsed wave Doppler of the aortic and mitral valves should be used to annotate the timing of events. Timing of the aortic valve closure may also be obtained from the TDI curves. ${ }^{44}$ An apical four-chamber view is used to obtain a clear image of the walls with minimal artifact. The transducer should be manipulated to align the wall of interest parallel to the ultrasound beam. The sector width and depth should be narrowed to just beyond the borders of the wall to obtain a high FR $(>180)$. The velocity scale (pulse repetition frequency, PRF) should be adjusted to avoid aliasing. Three-cycle analysis is more reproducible than single-cycle analysis in TD imaging, and a minimum of three cycles must be recorded for offline processing. ${ }^{1}$

Strain and SR values are generated during offline analysis. The parameters are derived from a sample area (segment). The size of the segment is set by the size of a specific region of interest (ROI) within the myocardial wall, which is determined by the operator (length and width) and a strain length (SL). The length of the ROI should be adequate for optimal calculation, while minimizing noise, and the width should not be larger than the width of the actual wall of interest. The operator must set the SL, also referred to as a computational distance. The SL is the length along the ultrasound beam against which the velocities for each point within the ROI are compared to derive the velocity gradient. The segment size will be larger than the ROI as it stretches parallel to the ultrasound beams toward the apex and the base of the heart.
The SL should not project outside the borders of the wall (into the atrial tissue for example; Fig. 6). Additional "optional" settings, i.e., Gaussian smoothing and drift compensation, can be utilized to minimize noise; however, with good image quality these settings are most likely not necessary (Fig. 7). Optimal probe choices, ideal ROI width and length and SL lengths have been published for term and preterm infants and are summarized in Table 1.35,36,38,39,43

Reference ranges and clinical applicability in the neonatal population

Reference ranges for deformation parameters exist in the term and preterm neonates ${ }^{26,35,37,45}$ (Table 2). In the term population, LV longitudinal strain values range between -20 and $-25(\%)$, while SRs values range between -1.5 and $-2.51 / \mathrm{s}$, SRe between 2.8 and 3.2 1/s, and SRa slightly lower between 2.1 and $2.41 / \mathrm{s}$. The RV free wall has higher strain values when compared with LV free wall and septum; however, SR values are comparable. The differences between LV and RV strain values may reflect the differing loading conditions between the RV and LV in the early neonatal period that may have an impact on strain but not SR. HR and the persistence of fetal shunts during the early transitional period appear to have a negligible impact on the measurements, but age may play a role in the first year of age. ${ }^{11}$

There is early evidence of the utility of deformation parameters in several disease states in term infants. Nestaas et al. demonstrated that LV and RV deformation parameters are uniformly lower in infants with hypoxic ischemic encephalopathy (HIE) compared with healthy controls. ${ }^{35}$ Interestingly, these differences occurred while SF was preserved between groups, further demonstrating that strain imaging permits a more comprehensive assessment of myocardial performance in neonates that could not be previously obtained with conventional imaging. ${ }^{35}$ The same group of investigators also demonstrated that infants with HIE have similarly impaired myocardial function during days 1-3, irrespective of whether they received therapeutic hypothermia, suggesting that myocardial injury may be a result of the initial insult rather than "cooling" treatment. ${ }^{46}$ In term infants with severe persistent pulmonary hypertension of the newborn (PPHN) not responsive to inhaled nitric oxide, RV strain significantly improves following the administration of milrinone over a 24-h period. ${ }^{47}$ With the establishment of reference values, TD-derived

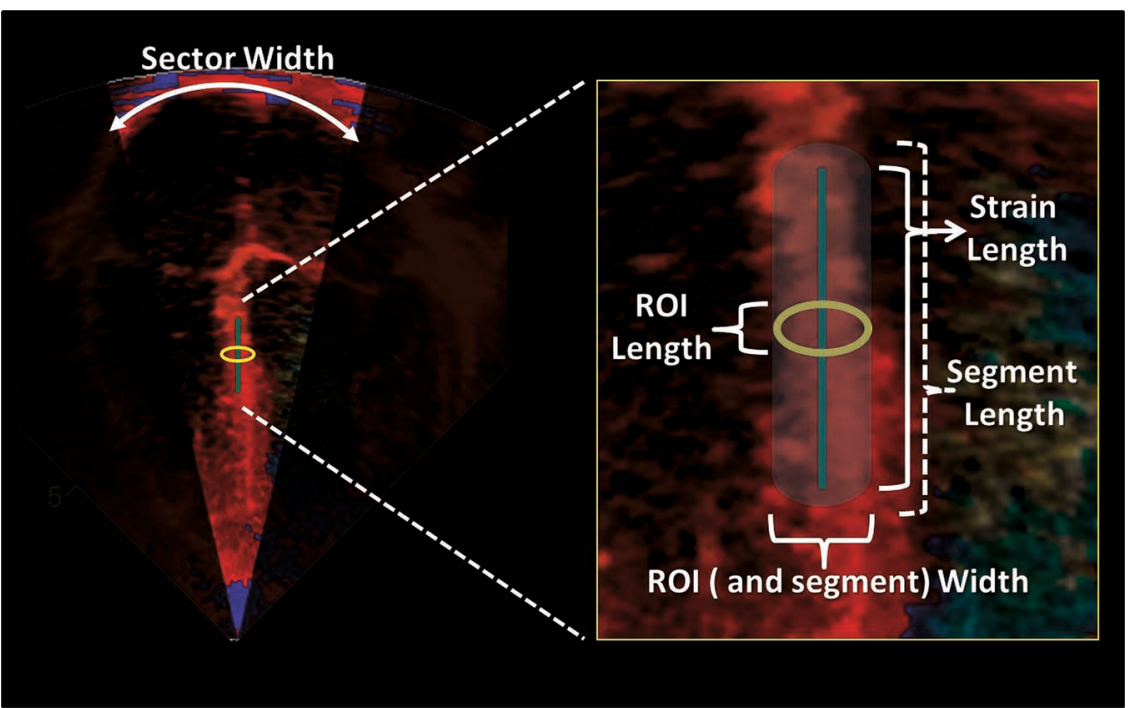

Fig. 6 Offline measurement of SR and strain using tissue Doppler. The sector width should be narrowed to increase the frame rate. The basal segment of the wall is usually interrogated to obtain SR and strain values. The ROI dimensions (length and width) are set by the operator. Strain length is also set while ensuring that the borders of the segment are not in contact with artifact or atrial tissue. The ROI can be moved slightly along the wall to obtain a clean and noise-free SR and strain curve (see Fig. 7) 
deformation measures can now be used to assess the efficacy of patient management strategies in health and disease and monitor treatment response.

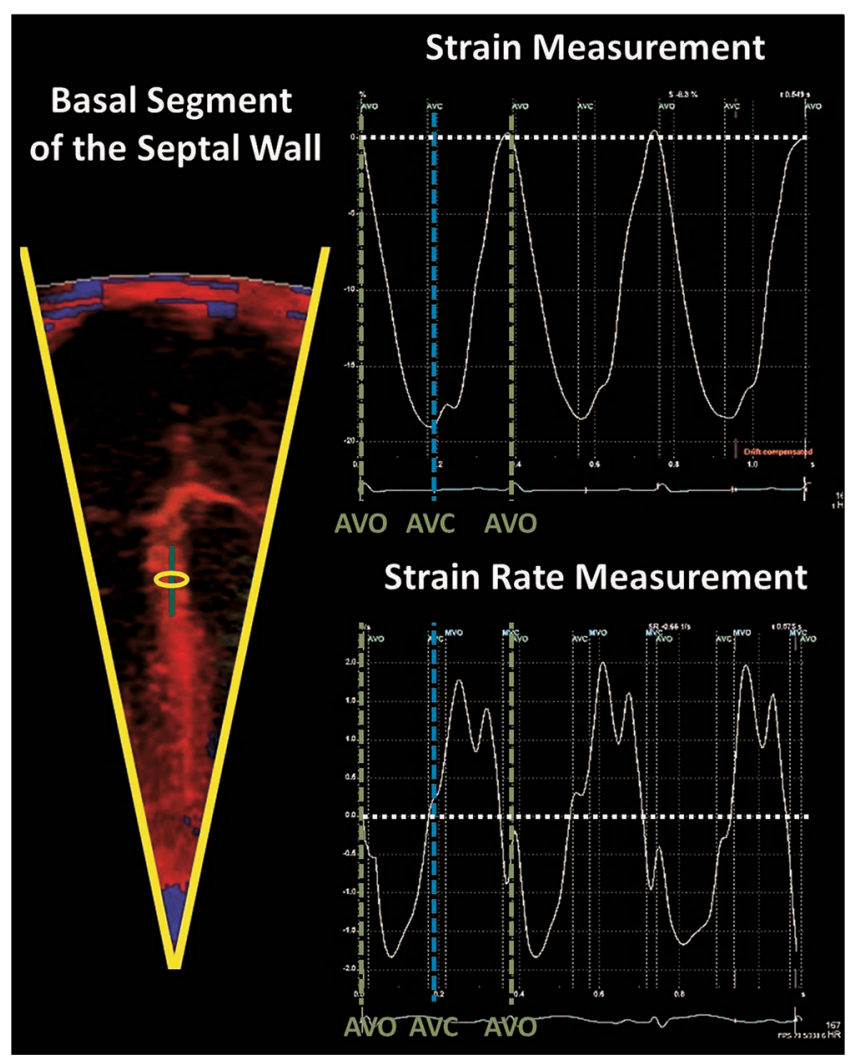

Fig. 7 An example of clear and artifact-free strain and strain rate curves over three cardiac cycles. Note the timing of events within the cardiac cycle. Strain peaks at end-systolic at aortic valve closure (AVC) and systolic strain rate peaks in mid-systole between aortic valve opening (AVO) and AVC

Table 1. Optimal setting for tissue Doppler deformation measurement

\begin{tabular}{lll}
\hline & Term infants & Preterm infants \\
\hline Probe & $5 \mathrm{~s}$ or $7 \mathrm{~s}$ & $10 \mathrm{~s}$ or $12 \mathrm{~s}$ \\
Sector width & Narrow & Narrow \\
Sector depth & Shallow & Shallow \\
Velocity scale (cm/s) (avoid aliasing) & -16 to 16 & -16 to 16 \\
Transducer frequency $(\mathrm{MHz})$ & $2.5-3.0$ & 8.0 \\
Pulse repetition frequency $(\mathrm{kHz})$ & 1.0 & 2.0 \\
Velocity scale (cm/s) & 16 & 16 \\
Frame rate (FPS) & $>200$ & $>200$ \\
Region of interest length $(\mathrm{mm})$ & 1 & 1 \\
Region of interest width $(\mathrm{mm})$ & 2 or 3 & 2 or 3 \\
Strain length (mm) & $10-20$ & 6 \\
Linear drift compensation & On & On \\
Gaussian smoothing & On & On \\
\hline
\end{tabular}

These settings apply for the General Electric Vivid scanners and Echo Pac Software (GE Medical, Milwaukee, USA). These recommendations are a guide only and setting may differ depending on new information emerging
In the preterm population, transitional strain and SR values are uniformly lower than those of term infants (Table 2). Two studies have examined the maturational changes of basal deformation parameters over the first few weeks of age. ${ }^{26,34}$ LV free wall strain and SR values remain stable over the first week of age with LV global strain showing a slight increase by 36 weeks postmenstrual age (PMA). Septal and RV free wall show a steadier increase over the first week of age and through 36 weeks PMA. ${ }^{26,34}$ Like term infants, weight, gestation, and HR have a minimal impact on these parameters. However, in the early transitional period, there is a negative correlation between echocardiography-derived estimates of SVR and LV and septal strain values, and a positive correlation between increasing preload associated with a PDA and LV strain. ${ }^{26,34}$ The relationship between SR values and cardiac loading measures were less pronounced, further supporting the dominant load dependency of strain but not SR. Finally, infants with chronic lung disease (CLD) have lower RV strain $(-26.4$ vs. $-30.7 \%, p=0.0 .1)$ and RV SRa [4.2 vs. $5.31 / \mathrm{s}, p=0.04)$ independent of gestation. CLD was shown to be associated with increased pulmonary arterial pressure, which may explain this association. ${ }^{34}$

\section{TWO-DIMENSIONAL}

\section{SPECKLE TRACKING ECHOCARDIOGRAPHY}

Principles and validation in the neonatal population

Two-dimensional STE (2D STE) is an imaging technique that uses standard B-mode images to measure deformation by tracking the movement of speckles within the myocardial wall. Speckles represent fixed tissue markers, or "natural acoustic markers," that are randomly distributed throughout the myocardium and have their own unique signature or "fingerprint". ${ }^{20}$ The speckle patterns result from acoustic backscatter generated by the reflected ultrasound beam. The movement of this speckled pattern follows myocardial tissue motion as it tracks the defined region of speckles, frame by frame and eventually over the entire heart cycle deriving the following information from segments of the myocardial wall: displacement (the movement of those speckles), velocity (the speed at which this movement occurs), strain (the relative change in distance between those speckles), and SR (the speed at which the change in distance occurs; ${ }^{9}$ ).

Specialized measuring software programs divide the myocardial walls of interest into segments and generate strain and strain rate values for each region (Fig. 8). Both regional and global functional parameters can be derived using this 2D STE deformation. 2D STE is angle independent within the ultrasound sector allowing the software to track the speckles in any direction. ${ }^{48}$ This angleindependency is the major advantage of 2D STE over TD-derived deformation, as the alignment of the wall relative to ultrasound beam is not necessary. With this relative freedom, enhanced imaging of the myocardial walls is possible. This is of particular importance to the LV free wall, as it can now be imaged at an angle to avoid lung artifact. However, 2D STE employs relatively lower FRs than TD-derived deformation (80-120 frames/s vs. $>200$ frames/s) and SR parameters, which rely on high temporal resolution, may not be as easy to interpret as strain, particularly in preterm infants with high HRs. Circumferential deformation is more prone to under sampling due to low FRs when compared with longitudinal deformation; ${ }^{49}$ generally, under sampling is avoided by a FR/HR ratio above 1 frame per second/beat per minute (in vitro model). Although 2D STE is less influenced by artifact, it remains highly reliant on clear imaging of the walls without dropouts. ${ }^{50}$

2D STE strain imaging is feasible in neonates with over $85 \%$ of acquisitions deemed as adequate quality to analyze.,3,11,32 In preterm infants, the reproducibility of LV global longitudinal strain (LV GLS) is high with intra- and inter-observer ICC values of 0.92 and 0.93 , respectively, and Bland-Altman analysis showing no significant bias between observers, with good agreement. ${ }^{2,11}$ de 
Table 2. Reference ranges for tissue Doppler deformation parameters in the neonatal population

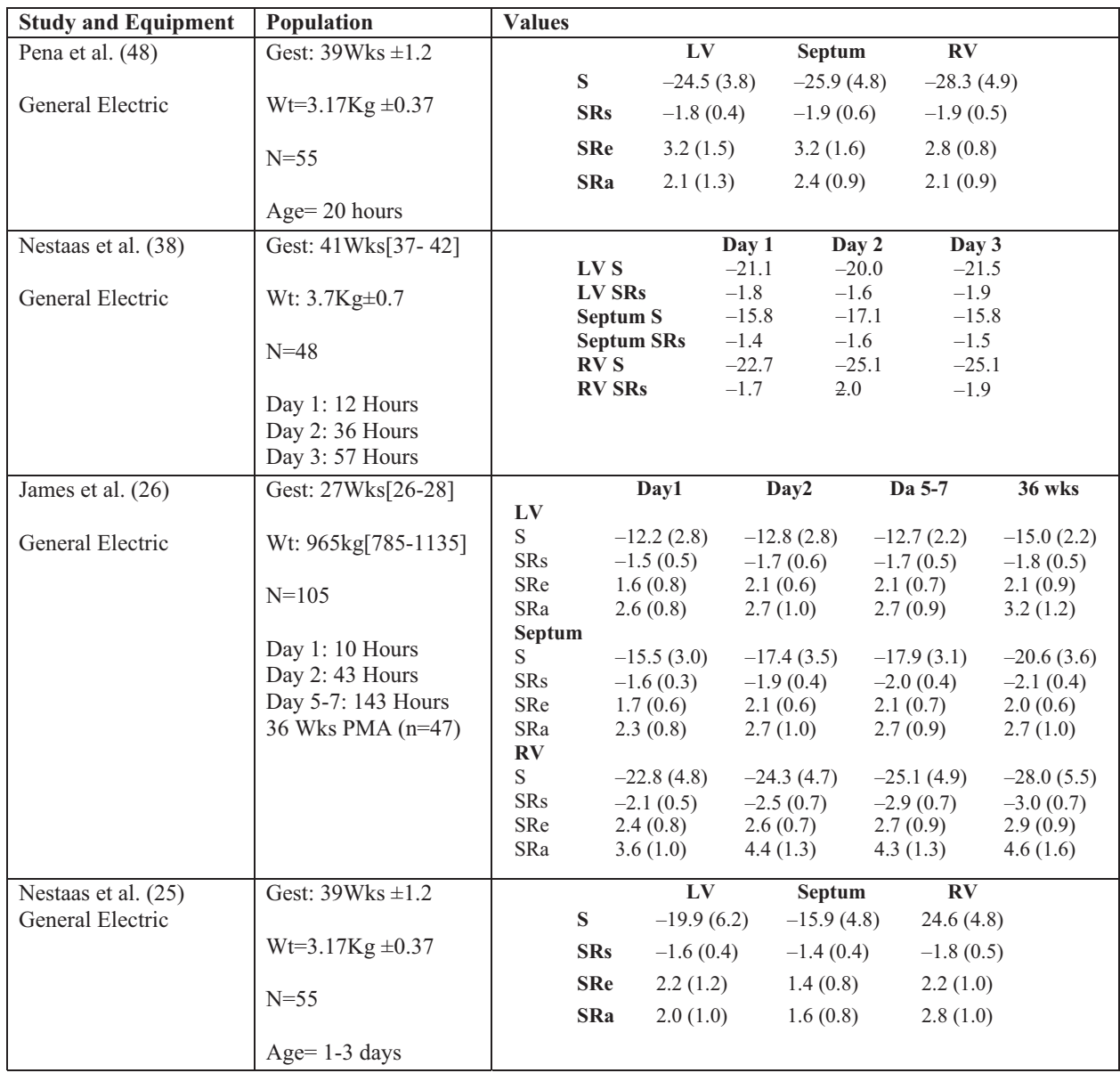

Values presented as means and standard deviations if available. All values are obtained from the basal segments in the four-chamber view. All strain units are $\%$, strain rate $1 / \mathrm{s}$

Gest gestation, $N$ number of infants, Wt birthweight, Wks weeks, $L V$ left ventricle, $R V$ right ventricle, PMA post-menstrual age, $S$ strain, $S R s$ systolic strain rate, SRe early diastolic strain rate, $S R a$ late diastolic strain rate (during atrial contraction)

Waal et al. also reported that LV circumferential strain was highly reproducible with intra- and inter-observer ICC $>0.85$ and COV $<10 \%$, but radial strain demonstrated very poor reproducibility with COV values between 18 and 50\%. ${ }^{19,32}$ With RV-focused imaging, there is a high degree of intra-observer (bias 3\%; COV 2.7\%; ICC 0.97) and inter-observer (bias 7\%; COV 3.9\%; ICC 0.93; $p$ $<0.05$ ) agreement for RV longitudinal strain. ${ }^{3}$ In term infants, Jain et al. demonstrated that LV and RV longitudinal strain measurements are highly reproducible with ICCs $>0.9$ and COVs $<10 \% .{ }^{10,51}$ Recently, Nestaas et al. demonstrated that the intra- and interobserver ICC for longitudinal peak systolic strain and SR were all above 0.87 for LV and RV analysis. ' Septal strain and SR from a nine-segment model of the three apical views also have moderate reproducibility measures. ${ }^{11}$

Image acquisition and offline measurement

Regional and global LV longitudinal deformation parameters are obtained from the apical four-, two-, and three-chamber views (Fig. 9). LV circumferential, radial, and rotational deformation are obtained from the parasternal short axis view at the level of the mitral valve (base), papillary muscles (mid-ventricular), and the apex. ${ }^{50}$ RV longitudinal deformation is obtained from a focused RV four-chamber view $\left({ }^{3,18,52}\right.$; Fig. 10$)$, and has been shown to provide the most feasible and reproducible measure of RV longitudinal deformation analysis in neonates. ${ }^{3}$ A modified RV three- chamber view can also be used to obtain RV deformation parameters. This view allows for "capture" of the maximum RV cavity, and provides a more direct assessment of the RV-pulmonary vascular axis between the RV free wall and the pulmonary circulation through the RV outflow tract. ${ }^{10}$ Although, in theory, the septum can be regarded as bi-layered and contributing to function of both ventricles, it is currently regarded as part of LV function. ${ }^{53}$

For optimal results, the same principles of image acquisition described for TD-derived deformation apply to 2D STE. Echocardiographic evaluation can be acquired in the resting state without sedation and gray scale images need to illustrate walls clearly and without artifact. An ECG signal is also mandatory in addition to event timing annotation, as described earlier. Fundamental and harmonic imaging with different probe types have not shown differences in strain and SR values in an in vitro study. ${ }^{49}$ The choice of transducer should be based on the frequency, which obtains the highest quality images with clear speckles. 2D STE is angle independent so the transducer can be manipulated off plane to obtain the ideal image. Sanchez et al. demonstrated that in order to obtain optimal reproducibility results for longitudinal 

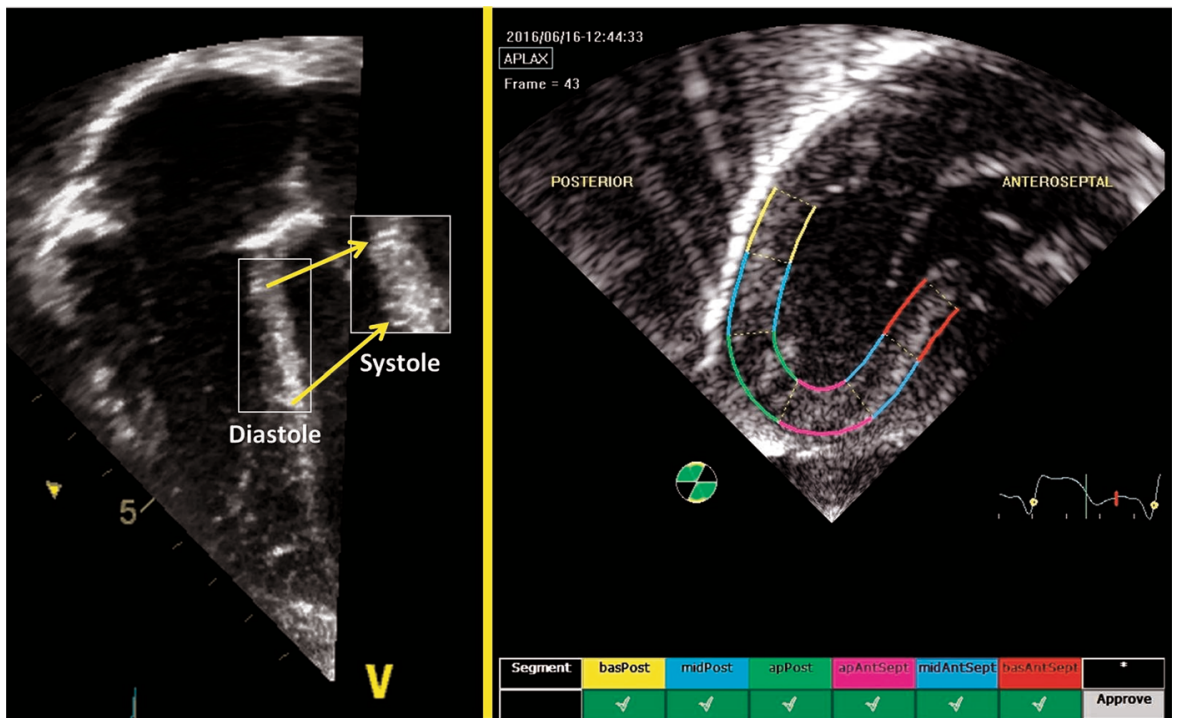

Fig. 8 2D Speckle Tracking Echocardiography. Speckles are acoustic back scatter that form a unique pattern within the myocardial walls. Those can be tracked throughout the cardiac cycle to derive deformation measurements. In this apical 3-chamber view of the LV, the myocardial walls are divided into segments and deformation parameters are presented individually for each segment to determine regional function. In addition, deformation for the whole region of interest is used to determine global function
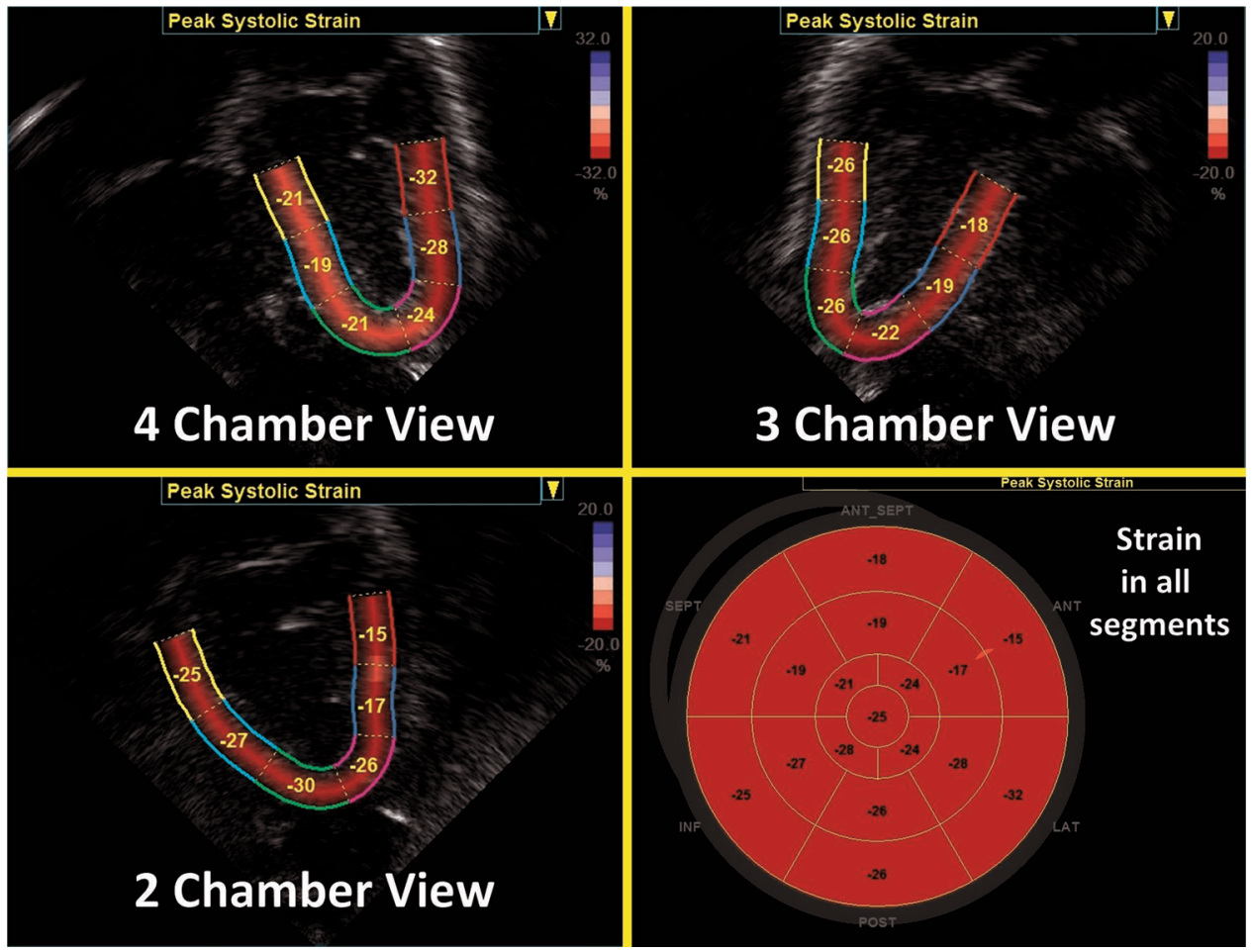

Fig. 9 Segmental strain in the three apical planes of the LV and a summary in a "Bullseye" pattern. Global longitudinal strain (often referred to as GLS) is the peak value in a compound curve made from the region of interest from the three planes

strain assessment, an FR to HR ratio of at least $0.7-0.9$ needs to be applied to the acquired images. Manipulations of depth and sector width can be used to achieve this ratio. ${ }^{54}$ Generally, an FR of $110-130$ is required for preterm infants and $90-110$ for term infants. $^{54}$ The images should be optimized to demonstrate the speckles and endocardial and epicardial borders clearly.

In neonates, analysis of deformation parameters is performed offline using dedicated vendor-customized analysis software. ${ }^{3}$ Newer packages of imaging and software systems allow for strain measurements to be assessed on the ultrasound machine in real time during image acquisition, but its feasibility and reliability have not been assessed in neonates. Vendorindependent software packages are available for speckle tracking analysis with any image acquisition platform. ${ }^{19}$ In some software packages, an ROI is defined by tracing the endocardial border of the myocardium at end-systole. The width of the ROI is set to match the width of the wall of interest (Fig. 8). The software then automatically tracks the movement of speckles to derive the deformation parameters. The acceptability of the tracking is automatically suggested by the software. The user 


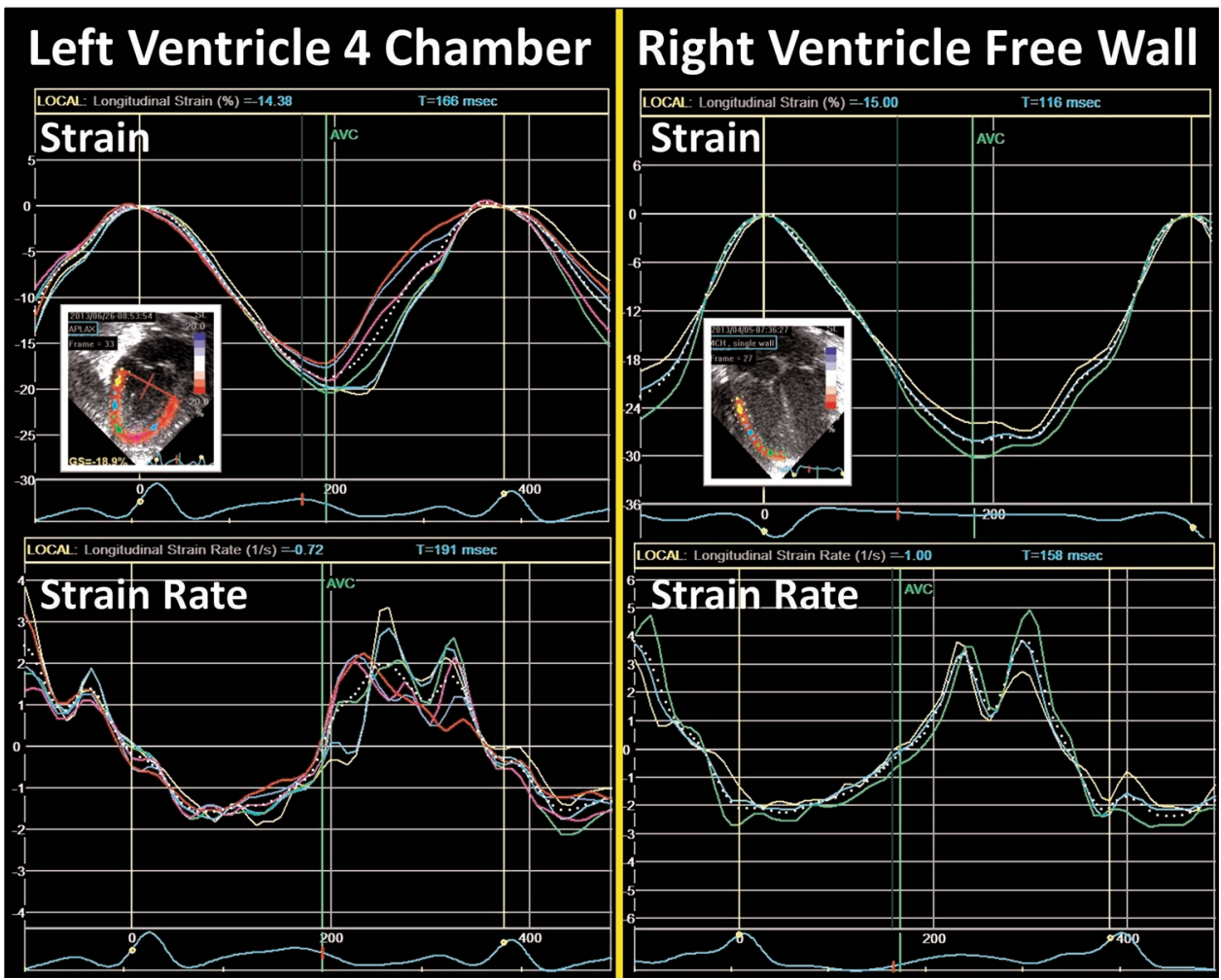

Fig. 10 Strain and strain rate curves from the LV four-chamber view and the RV free walls. The colored lines represent the deformation values from each segment and the dotted white line represents the values from the whole region of interest. Notice the relative increased level of noise in the strain rate curves (see text)

can also visually inspect the quality of the tracking before finally accepting or rejecting analysis of the segment. To enhance the STE capabilities, the ROI is readjusted repeatedly to avoid free wall base over excursion, tracking of the trabeculations, and avoid artifact. Cine-loop images with persistently inadequate tracking should be excluded from analysis. Once the integrity of myocardial STE is visually confirmed by the user, the software algorithm generates seven curves for each view of heart (i.e., apical four chamber, parasternal short axis view at the mitral valve, etc.) based on the timing of the opening and closure of the semilunar valves (aortic valve for LV strain and pulmonary valve for RV strain). Each curve represents the measured myocardial deformation (strain) for the six specific myocardial segments (basal septum, mid-septum, apical septum, basal lateral, mid-lateral, and apical lateral) and one global value representing the combined strain from all segments within the specific echocardiographic view (Fig. 10).

\section{REFERENCE RANGES AND CLINICAL UTILITY IN THE NEONATAL POPULATION}

Normative data and reference ranges are still emerging for deformation parameters obtained using 2D STE in the preterm and term infants. Reference ranges have been published in healthy uncomplicated term ${ }^{10,35,37,45,51,55-58}$ and preterm population, ${ }^{11,19,26,32,53,59,60}$ or reported results from control groups of neonates (who were recruited for specific studies; ${ }^{61-66}$ ). The process of standardization and reference values in neonates stems from relatively small number of infants included in each study, the varying time points at which echocardiograms were acquired in the first year of age, and the multitude of vendors and software versions utilized for acquisition and post-processing. In addition, only two studies have assessed true "global" LV longitudinal (from the three apical chamber views; ${ }^{11,51}$ ), while most have reported LV longitudinal strain values from the LV four-chamber. ${ }^{10,19,53,57,58,64,65}$ There are only two studies that report circumferential strain, and both only generate circumferential strain from the mid-ventricular (papillary muscle) level of the LV free wall. ${ }^{60,64}$ Table 3 summarizes the current available literature. Radial deformation values and diastolic SR parameters (early and atrial) measured using 2D STE remain unreliable in the neonatal population. ${ }^{26}$ In general, LV deformation parameters measured using 2D STE appear to remain stable during the transitional period and up to 28 days. ${ }^{19,26,65-67}$ RV strain parameters gradually increase beyond the transitional period and through the first year of age. ${ }^{1,26,59}$ Strain and SRs values are higher in the RV than the LV, reflective of the changing loading conditions specific to each ventricle. ${ }^{11,26}$ In the LV, circumferential deformation parameters appear to be slightly higher than longitudinal deformation. ${ }^{60,64}$

2D STE has also been examined in some disease states in neonates. ${ }^{2,27,61,63,66}$ One of the first studies of 2D STE in preterm infants illustrated the negative impact of PDA ligation on LV GLS in the immediate post-operative period, followed by recovery 24 $\mathrm{h}$ later. ${ }^{2}$ The reduction in LV GLS post-operatively was attributed to the increase in afterload and the decrease in preload associated with the procedure. In the early transitional period, another study demonstrated that the administration of antenatal magnesium sulfate is associated with a lower SVR and a higher LV GLS on day 1 of age. ${ }^{27}$ These studies further highlight the load dependency of strain. The influence of common cardiopulmonary abnormalities in preterm infants (such as CLD and pulmonary hypertension) appear to leave a negative effect on RV and septal strain, with preservation of LV strain patterns. ${ }^{11,61,65} \mathrm{LV}$ and RV function have also been evaluated in term infants of diabetic mothers (gestational and pre-gestational diabetes; ${ }^{63,68}$ ). LV GLS is lower in pre-gestational $(-10.4 \pm 3.2, n=20)$ and gestational $(-13.1 \pm 4.7, n=25)$ groups when compared with the control 
Table 3. Reference ranges for speckle tracking echocardiography deformation parameters in the neonatal population

\begin{tabular}{|c|c|c|c|c|c|c|c|}
\hline Study and Equipment & Population & \multicolumn{6}{|l|}{ Values } \\
\hline General Electric & $\begin{array}{l}\text { Gest: } 40 \mathrm{Wks} \pm 1.2 \\
\mathrm{Wt}=3.5 \mathrm{Kg} \pm 0.5 \\
\mathrm{~N}=28 \\
2 \text { Days } \\
23 \text { Days } \\
48 \text { Days }\end{array}$ & $\begin{array}{l}\mathrm{LV} 4 \mathrm{Ch} \\
\mathrm{LV} \text { paras }\end{array}$ & $\begin{array}{l}\text { amber view or } \\
\text { ternal short a }\end{array}$ & $\begin{array}{l}\text { 1-3 Days } \\
-18.8 \pm 3.5 \\
-19.7 \pm 4.3\end{array}$ & $\begin{array}{l}3 \text { Weeks } \\
-18.6 \pm 2.5 \\
-20.5 \pm 4.5\end{array}$ & s) fo & $\begin{array}{l}\text { - } 7 \text { Weeks } \\
19.4 \pm 2.3 \\
19.6 \pm 4.9\end{array}$ \\
\hline Schubert et al. (63) & $\begin{array}{l}\text { Gest: Term } \\
\mathrm{Wt}=\text { No SGA } \\
\mathrm{N}=30 \\
170 \text { hours }[135-207]\end{array}$ & $\begin{array}{l}\text { Longitu } \\
\text { Systolic } \\
\text { *Basal V }\end{array}$ & $\begin{array}{l}\text { Idinal Strain } \\
\text { Strain Rate* } \\
\text { alues Presented }\end{array}$ & $\begin{array}{c}\mathbf{L V} \\
-19.5 \pm 2.1 \\
-2.94 \pm 1.0 \\
\text { Refer to man }\end{array}$ & $\begin{array}{r}\text { Septum } \\
1 \quad-19.5 \pm 2 . \\
5 \quad-2.23 \pm 0 . \\
\text { nuscript for fur }\end{array}$ & 17 & $\begin{array}{c}\mathbf{R V} \\
-23.0 \pm 4.3 \\
-3.61 \pm 1.59 \\
\text { details }\end{array}$ \\
\hline $\begin{array}{l}\text { Jain et al. }(10,61) \\
\text { General Electric }\end{array}$ & $\begin{array}{l}\text { Gest: } 40 \mathrm{Wks} \pm 1.2 \\
\text { Wt: } 3.49 \mathrm{Kg} \pm 0.44 \\
\mathrm{~N}=50 \\
\text { Day 1: } 15 \text { Hours } \\
\text { Day 2: } 35 \text { Hours }\end{array}$ & $\begin{array}{l}\text { RV para } \\
\text { LV paraı }\end{array}$ & $\begin{array}{l}\text { RV 4-Chamt } \\
\text { RV 3-Chamb } \\
\text { RV Global S } \\
\text { LV Global S } \\
\text { LV Global S } \\
\text { * Early and la }\end{array}$ & $\begin{array}{l}\text { er Strain } \\
\text { er Strain } \\
\text { rain } \\
\text { rain } \\
\text { ss } \\
\text { te LV SR in ( }\end{array}$ & $\begin{array}{c}\text { Day } 1 \\
-21.2 \pm 5.3 \\
-21.4 \pm 4.4 \\
-21.2 \pm 3.9 \\
-21.7 \pm 1.9 \\
2.05 \pm 2.0 \\
61)\end{array}$ & $\begin{array}{r}\mathbf{D} \\
-21 \\
-20 \\
-21 \\
-21 \\
2.1\end{array}$ & $\begin{array}{l}\text { ay } 2 \\
.3 \pm 5.4 \\
.6 \pm 4.2 \\
.2 \pm 4.2 \\
.2 \pm 1.8 \\
7 \pm 2.3\end{array}$ \\
\hline Nasu et al (56) & Gest: $33 \mathrm{Wks} \pm 2$ & $4 \mathrm{Chamb}$ & er View Used & Septum was & s divided into & LV & and RV sides \\
\hline QLAB, Philips & $\begin{array}{l}\mathrm{Wt}=1.9 \mathrm{Kg} \pm 0.21 \\
\mathrm{~N}=21 \\
\text { Serial Data }\end{array}$ & $\begin{array}{l}\text { LV } \\
\text { Sep-LV } \\
\text { RV } \\
\text { Sep-RV } \\
\text { More tin }\end{array}$ & $\begin{array}{c}\mathbf{1} \mathbf{~ H r} \\
-23.8 \pm 4.0 \\
-22.7 \pm 5.7 \\
-22.0 \pm 4.7 \\
-17.9 \pm 4.5 \\
\text { e points avail }\end{array}$ & $\begin{array}{c}\mathbf{2 4} \mathbf{H r} \\
-23.4 \pm 3.2 \\
-21.0 \pm 5.1 \\
-24.7 \pm 4.7 \\
-17.7 \pm 4.6 \\
\text { bble in the M }\end{array}$ & $\begin{array}{r}\mathbf{4 8 ~ H r} \\
-23.8 \pm 5 . \\
-19.8 \pm 3 . \\
-20.8 \pm 2.6 \\
-17.3 \pm 4.5 \\
\text { Ianuscript. }\end{array}$ & & $\begin{array}{c}\mathbf{7 2} \mathbf{H r} \\
-24.9 \pm 3.2 \\
-20.9 \pm 3.0 \\
-22.9 \pm 5.1 \\
-14.7 \pm 4.6\end{array}$ \\
\hline
\end{tabular}


Table 3 continued

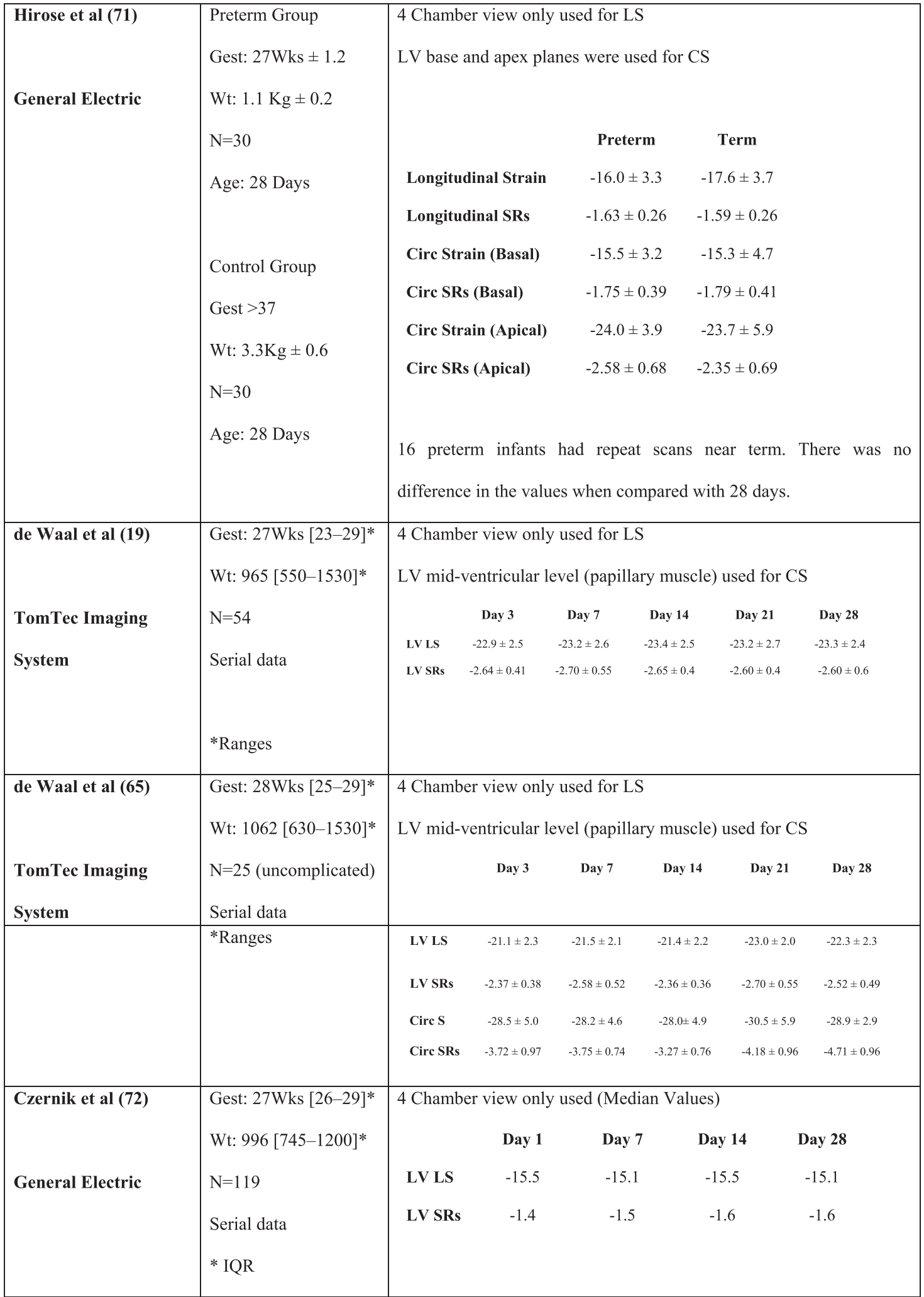




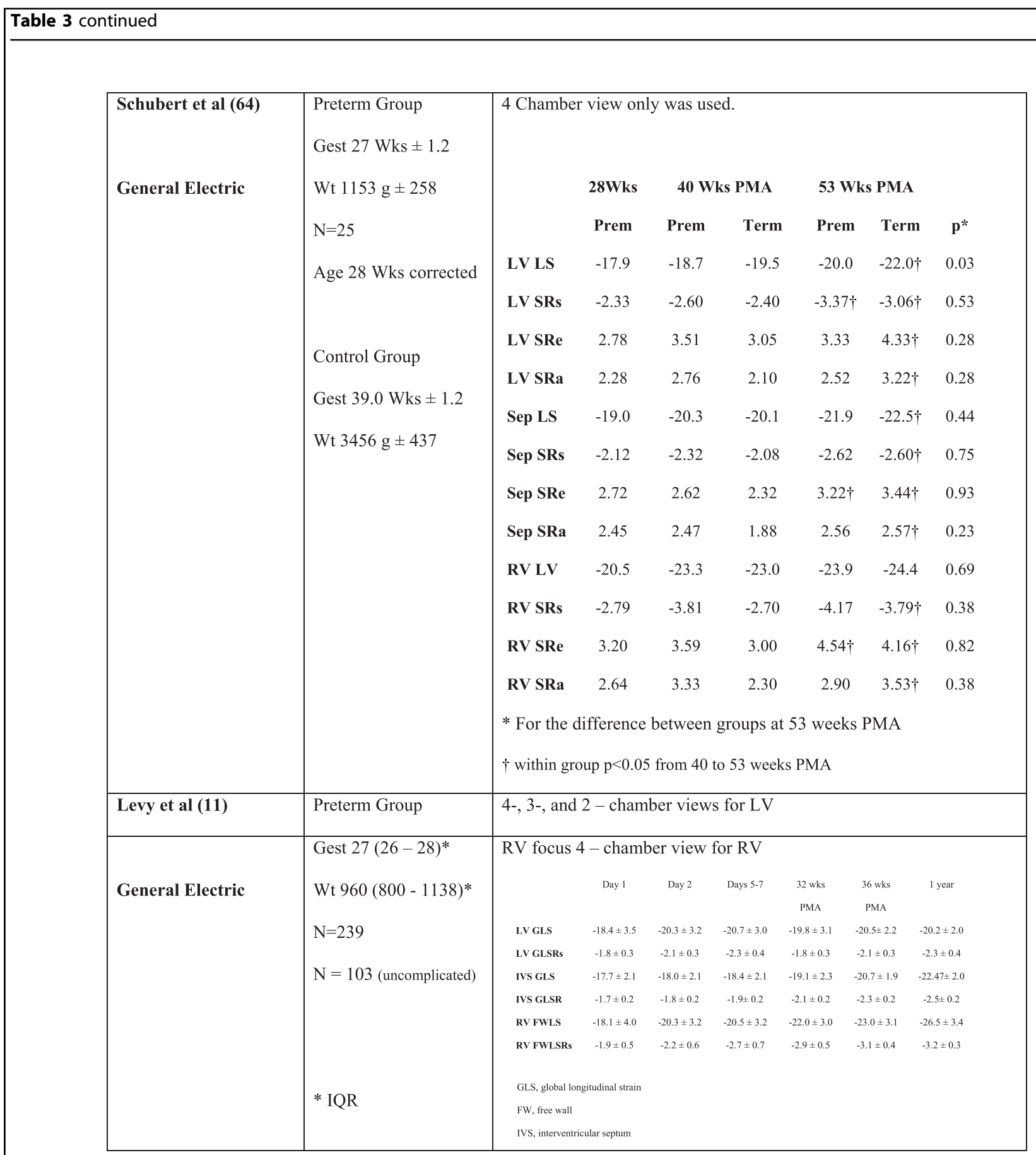

Values presented as means and standard deviations if available, unless stated otherwise. All strain units are $\%$ and strain rate $1 / \mathrm{s}$

Gest gestation, $N$ number of infants, $W t$ birthweight, $W$ ks weeks, $L V$ left ventricle, $R V$ right ventricle, Sep septum, $P M A$ post-menstrual age, Circ circumferential, $L S$ longitudinal strain, $S R s$ systolic strain rate, SRe early diastolic strain rate, $S R a$ late diastolic strain rate

group $(-19 \pm 2, n=45)(p<0.01) .{ }^{63}$ Similarly, LV GLS can identify dysfunction in severely asphyxiated term infants who are undergoing therapeutic hypothermia when compared with healthy controls $(-11.01 \% \pm 2.48$ vs. $-21.45 \% \pm 2.74, p<$ $\left.0.001 ;{ }^{66}\right)$. LV GLS has a significant correlation with troponin levels $\left(r^{2}=0.64, p<0.001\right)$ suggesting that LV GLS is also capable of grading disease severity. ${ }^{66}$ Finally, 2D STE strain was found to be significantly lower in term infants with proven sepsis in the first month of age when compared to age- and weightmatched controls.

\section{ROTATIONAL MECHANICS}

Principles and validation in the neonatal population

Myocardial shear deformation in the circumferential-longitudinal plane results in torsional deformation of the LV during ejection and is utilized to characterize functional changes in systole and diastole. ${ }^{69}$ The complex architecture of the LV myocardium results in inhomogeneous contraction patterns. The myofiber orientation changes continuously from a right-handed helix in the subendocardium to a left-handed helix in subepicardium, enabling the LV to have unique rotational properties. ${ }^{50}$ The LV base rotates in a 

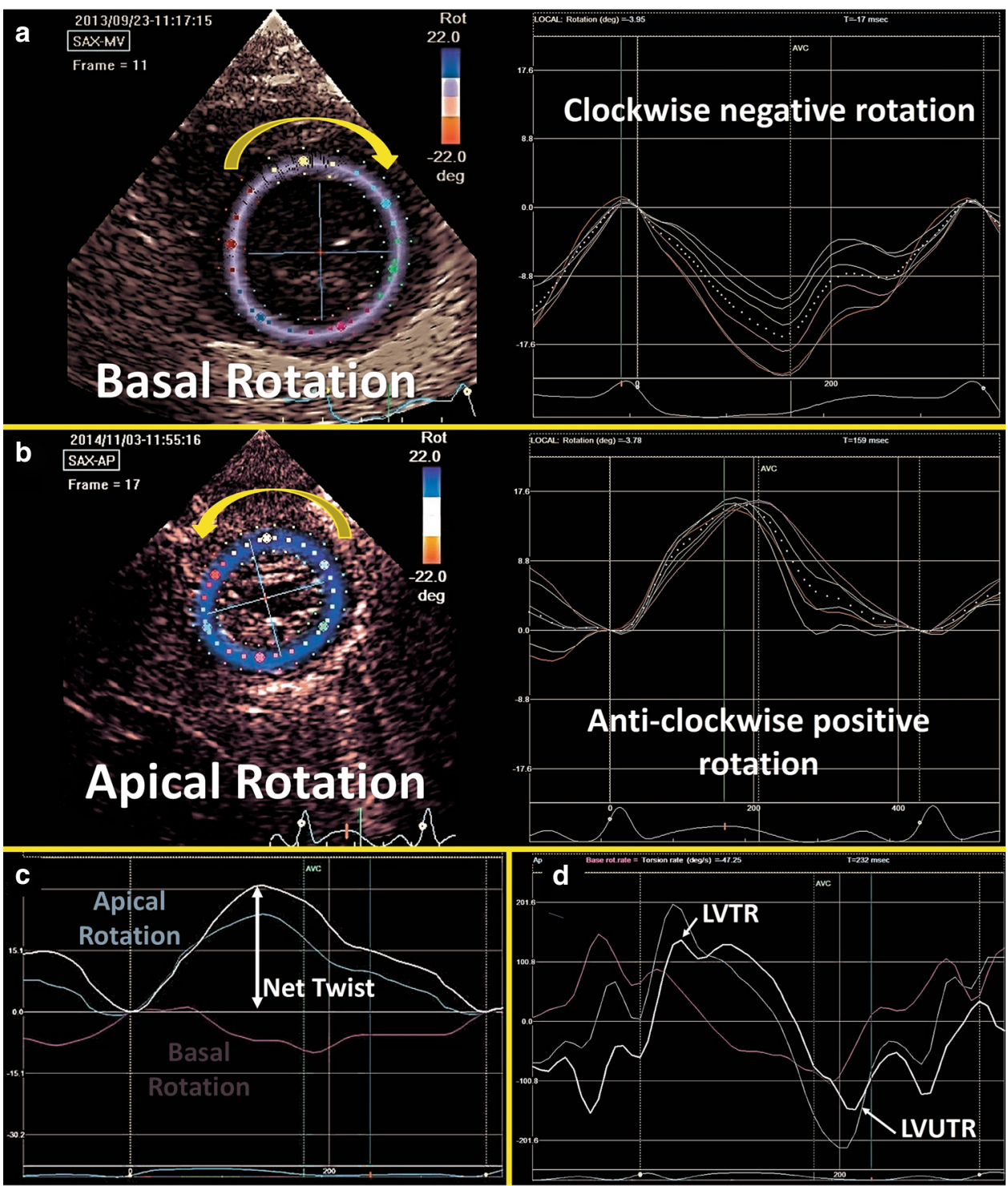

Fig. 11 Left ventricle rotational mechanics. a Basal rotation occurs in a clockwise direction (negative) and b Apical rotation occurs in an anticlockwise direction (positive). c The net effect of the opposing rotations is called Twist. $\mathbf{d}$ The speed at which twist occurs is called twist rate (LVTR) and the speed at which untwist occurs is called untwist rate (LVUTR)

clockwise direction (displayed as negative rotation in degrees, Fig. 11a) and the apex rotates in a counter clockwise direction (displayed as positive rotation in degrees. Figure $11 \mathrm{~b} ;{ }^{14}$ ). Twist (degrees) is defined as the difference between apical and basal systolic rotation (Fig. 11c), and the net effect of this phenomenon is an LV wringing motion that improves the ability of the LV to eject blood during systole. Torsion $(\% \mathrm{~cm})$ is the term used to describe LV twist indexed to its LV end-diastolic length (LV length is measured from the apical four-chamber view in diastole by measuring the distance between the midpoint of the mitral valve annulus and the apex) and enables the comparison of LV twist across differing LV sizes. The temporal derivative of twist is referred to as twisting and untwisting rate $\left(\% / s ;{ }^{21}\right)$. LV twist rate (LVTR) is the velocity at which twist occurs per unit time and is depicted as a positive value $(\% / s)$. Untwisting is the motion opposite to the direction of twist occurring in diastole. The speed at which LV untwist occurs is termed LV untwist rate (LVUTR; Fig. 11d). During diastole, the LV untwists to return to its baseline un-deformed and untwisted shape. The act of untwisting also aids in diastolic function and contributes to early diastolic filling. This process is highly dependent on the elasticity of the $\mathrm{LV}^{70} \mathrm{LV}$ untwist is facilitated by the kinetic energy stored during twisting in systole and therefore, LVUTR is highly dependent on LVTR (an example of systolic-diastolic interdependence). ${ }^{12}$ Like natural strain, increasing afterload appears to decrease LV twist and untwist rate in experimental animal models and human adults. ${ }^{71}$ Similarly, in preterm infants increased afterload appears to negatively impact these measurements. ${ }^{27}$ LVUTR also appears to be negatively influenced by increasing afterload, as it is highly dependent on LV twist. ${ }^{12}$ Ramani et al. demonstrated that basal LV rotation was reduced with preserved LV apical rotation in adult patients with pulmonary arterial hypertension. $^{72}$

There is a small, yet growing literature on the validation of rotational mechanics in neonates. ${ }^{12,63,73-75}$ Although the recommendations of European Association of Cardiovascular Imaging, EACVI, and the American Society of Echocardiography (ASE) task force to Standardize Deformation Imaging urge caution in the use of twist and torsion because both parameters are poorly defined in 2D echocardiography, recent work has demonstrated the improving feasibility and reproducibility analysis of rotational mechanics in neonates. ${ }^{12,73-75}$ In preterm infants, James et al. 
demonstrated acceptable intra- and inter-observer reliability with ICC ranging from 0.70 to $0.89 .^{12}$ Zhang et al. demonstrated that inter-operator COV was $\leq 5 \% .^{73}$ Al-Naami does not report reproducibility statistics, but comments that imaging was feasible in all subjects. ${ }^{75} \mathrm{Kim}$ et al. reported slightly lower feasibility at $80 \%$ with ICC $>0.9$ for all parameters. ${ }^{74}$

Image acquisition and offline measurement

Two-dimensional STE method is also used to derive rotational parameters. The methodology for image acquisition and offline assessment is similar to deformation assessment described above. Two-dimensional grayscale images of the LV base (at the level of the mitral valve leaflets) and apex (distal to the papillary muscles) are acquired from LV parasternal short-axis view. Image acquisition at the two planes of interest is carried out to ensure that the LV cross-section is as circular as possible. A protocol for rotational mechanics imaging and post-processing data analysis exists for $2 \mathrm{D}$ STE in neonates. ${ }^{12}$

Reference ranges and clinical utility in neonates

There is a paucity of data on LV rotational physiology in neonates. There are several age-related studies that have assessed the changes in rotational mechanics from birth through early adolescents, which also include a small subset of neonates. ${ }^{73-76}$ There are a few singlestudy reports that define control cohorts to compare with specific diseases in term neonates. ${ }^{63,68,77,78}$ Al-Biltagi et al. ${ }^{63}$ showed that cardiac torsion was impaired in infants of diabetic mothers (IDMs), and Liao et al. ${ }^{68}$ demonstrated a similar decrease in torsion, but with persevered $\mathrm{EF}$, suggesting that rotational mechanics may offer a more sensitive measure of ventricular function. Xie et al. evaluated the utility of torsion analysis in the assessment of infants with congenital heart disease. ${ }^{77}$

Rotational mechanics was recently studied in late preterm [mean (SD) gestation of $36.0( \pm 1.5), n=31]^{27}$ and extreme preterm infants [mean (SD) gestation of 26.8 weeks $( \pm 1.5), n=51]^{12}$ In extreme preterm infants, apical rotation remains constant over the first week of age $\left[11.8^{\circ}( \pm 5.0)\right.$ vs. $12.1^{\circ}( \pm 6.1)$ vs. $11.7^{\circ}( \pm 8.3)$, on days 1 , 2 , and $5-7 ; p=0.92]$. Basal rotation, however, changes from counter clockwise on day 1 and 2 to clockwise on day 7 [5.5 [ -0.3 to 8.3$]$ vs. 4.0 [ -4.7 to 7.2$]$ vs. -4.5 [ -5.8 to -2.3$], p<0.001]$ with a resultant net increase in twist, torsion, and LVUTR. ${ }^{12}$ Future studies are now required to determine the clinical relevance of rotational mechanics, the effects of various disease states (cardiomyopathies, chronic cardio-respiratory disease, sepsis, etc.), explore the relationships to conventional echocardiographic measures, and to determine reference ranges in preterm and term infants.

\section{CONCLUSION}

The assessment of deformation parameters to properly characterize cardiac function in neonates has gained considerable interest. The emerging literature continues to demonstrate the feasibility and reproducibility of strain values derived by both TDI and 2D STE in the neonatal (term and preterm) population, and the relative advantages of those techniques when compared to conventional measures. With the establishment of reference ranges and normative data, the routine clinical use of strain, SR, and rotational mechanics is likely to become more common. As the clinical applicability of these measures is further elucidated in neonates, we will begin to understand their ability to direct management, monitor treatment response, and predict outcomes to optimize the care delivered to neonates. Future work should focus on the ability of those measurements to distinguish between myocardial dysfunction secondary to adverse loading conditions and dysfunction resulting from impaired intrinsic contractility (or a mixture of both). This will help tailor the therapeutic interventions to more accurately target the underlying pathophysiological consequences of disease states.

\section{ACKNOWLEDGEMENTS}

All members of the European Special Interest Group 'Neonatologist Performed Echocardiography' are listed in the appendix. All these members have substantially contributed to the conception and revision of the manuscript and approved the final version to be published. A.E.-K. is in receipt of an Irish Health Research Board Clinical Trials Network Grant (HRB CTN 2014-10) and an EU FP7/2007-2013 grant (agreement no. 260777, The HIP Trial). E.N. received grant support from Research Council of Norway and Vestfold Hospital Trust. Financial support of publication costs by the European Society for Paediatric Research (ESPR) is gratefully acknowledged.

\section{ADDITIONAL INFORMATION}

Competing interests: A.G. owned equity in Neonatal Echo Skills and has received grant support from the American Heart Association. D.V.L. is in receipt of an EU FP7/ 2007-2013 (agreement no. 260777 the HIP trial). E.D. received lecture fees and consulting fees from Chiesi Pharmaceutical. K.B. received lecture fees from Chiesi Pharmaceutical. M.B. holds a patent "Thermal shield for the newborn baby". S.G. received grant support from National Institute of Health Research, Health Technology Assessment (11/92/15), UK. S.R. received lecture fees for Phillips Ultrasound and GE Ultrasound. W.P.B. has received grant support from The Netherlands Organization for Health and Development (ZonMw; grant numbers 843002622 and 843002608 ). Z.M. has received lecture fees from Chiesi Pharmaceutical. The remaining authors declared no competing interests.

Publisher's note: Springer Nature remains neutral with regard to jurisdictional claims in published maps and institutional affiliations.

\section{APPENDIX}

European Special Interest Group 'Neonatologist Performed Echocardiography' (NPE), endorsed by the European Society for Paediatric Research (ESPR) and European Board of Neonatology (EBN)

de Boode W. P. (chairman), Department of Neonatology, Radboud University Medical Center, Radboud Institute for Health Sciences, Amalia Children's Hospital, Nijmegen, The Netherlands (willem.deboode@radboudumc.nl)

Austin T., Department of Neonatology, Rosie Hospital, Cambridge University Hospitals NHS Foundation Trust, Cambridge, United Kingdom (topun.austin@ addenbrookes.nhs.uk)

Bohlin K., Department of Neonatology, Karolinska University Hospital, Karolinska Institutet, Stockholm, Sweden (kajsa.bohlin@ki.se)

Bravo M. C., Department of Neonatology, La Paz University Hospital, Madrid, Spain (mcarmen.bravo@salud.madrid.org)

Breatnach C. R., Department of Neonatology, The Rotunda Hospital, Dublin, Ireland (colm.breatnach@gmail.com)

Breindahl M., Karolinska University Hospital, Karolinska Institutet, Stockholm, Sweden (morten.breindahl@sll.se)

Dempsey E., INFANT Centre, Cork University Maternity Hospital, University College Cork, Ireland (g.dempsey@ucc.ie)

El-Khuffash A., Department of Neonatology, The Rotunda Hospital, Dublin, Ireland; Department of Pediatrics, The Royal College of Surgeons in Ireland, Dublin, Ireland (afifelkhuffash@rcsi.ie)

Groves A. M., Division of Newborn Medicine, Mount Sinai Kravis Children's Hospital, New York, NY, USA (alan.groves@mssm.edu)

Gupta S., University Hospital of North Tees, Durham University, Stockton-on-Tees, United Kingdom (samir.gupta@nth.nhs.uk)

Horsberg Eriksen B., Department of Pediatrics, Møre and Romsdal Hospital Trust Ålesund, Norway (beate.eriksen@me.com)

Levy P. T., Department of Pediatrics, Washington University School of Medicine, Saint Louis, MO, USA; Department of Pediatrics, Goryeb Children's Hospital, Morristown, NJ, USA (Levy_P@kids.wustl.edu)

McNamara P. J., Departments of Pediatrics and Physiology, University of Toronto, Toronto, ON, Canada (patrick.mcnamara@sickkids.ca)

Molnar Z., John Radcliffe Hospital, Oxford, United Kingdom (zoltan.Molnar@ouh.nhs. uk)

Nestaas E., Institute of Clinical Medicine, Faculty of Medicine, University of Oslo, Norway; Department of Cardiology and Center for Cardiological Innovation, Oslo University Hospital, Rikshospitalet, Oslo, Norway; Department of Paediatrics, Vestfold Hospital Trust, Tønsberg, Norway (nestaas@hotmail.com)

Rogerson S. R., The Royal Women's Hospital, Parkville, VIC, Australia (sheryle. Rogerson@thewomens.org.au)

Roehr C. C., Department of Paediatrics, University of Oxford, John Radcliffe Hospital, Oxford, United Kingdom (charles.roehr@paediatrics.ox.ac.uk)

Savoia M, Azienda Ospedaliero-Universitaria S. Maria della Misericordia, Udine, Italy (marilena.savoia@gmail.com) 
Schubert U., Department of Clinical Science, Intervention and Technology, Karolinska Institutet, Stockholm, Sweden (ulfschubert@gmx.de)

Schwarz C. E., Department of Neonatology, University Children's Hospital of Tübingen, Tübingen, Germany (c.schwarz@med.uni-tuebingen.de)

Sehgal A., Department of Pediatrics, Monash University, Melbourne, Australia (arvind. sehgal@monash.edu)

Singh Y., Addenbrooke's Hospital, Cambridge University Hospitals NHS Foundation Trust, Cambridge, United Kingdom (yogen.Singh@nhs.net)

Slieker M. G., Department of Paediatric Cardiology, Radboudumc Amalia Children's Hospital, Nijmegen, The Netherlands (Martijn.Slieker@radboudumc.nl)

Tissot C., Department of Pediatrics, Clinique des Grangettes, Chêne Bougeries, Switzerland (cecile.tissot@hotmail.com)

van der Lee R., Department of Neonatology, Radboud University Medical Center, Radboud Institute for Health Sciences, Amalia Children's Hospital, Nijmegen, The Netherlands (Robin.vanderLee@radboudumc.nl)

van Laere D., Department of Pediatrics, Antwerp University Hospital UZA, Edegem, Belgium (david.VanLaere@uza.be)

van Overmeire B., Department of Neonatology, University Hospital Brussels, Brussels, Belgium (bart.van.overmeire@erasme.ulb.ac.be)

van Wyk L., Department of Paediatrics \& Child Health, University of Stellenbosch, Cape Town, South Africa (lizelle@sun.ac.za)

\section{REFERENCES}

1. Nestaas, E., Stoylen, A. \& Fugelseth, D. Speckle tracking using gray-scale information from tissue Doppler recordings versus regular gray-scale recordings in term neonates. Ultrasound Med. Biol. 42, 2599-2605 (2016).

2. El-Khuffash, A. F., Jain, A., Dragulescu, A., McNamara, P. J. \& Mertens, L. Acute changes in myocardial systolic function in preterm infants undergoing patent ductus arteriosus ligation: a tissue Doppler and myocardial deformation study. J. Am. Soc. Echocardiogr. 25, 1058-1067 (2012).

3. Levy, P. T., Holland, M. R., Sekarski, T. J., Hamvas, A. \& Singh, G. K. Feasibility and reproducibility of systolic right ventricular strain measurement by speckletracking echocardiography in premature infants. J. Am. Soc. Echocardiogr. 26, 1201-1213 (2013).

4. Ling, L. F. et al. Accuracy and interobserver concordance of echocardiographic assessment of right ventricular size and systolic function: a quality control exercise. J. Am. Soc. Echocardiogr. 25, 709-713 (2012).

5. Hoffmann, R. et al. Analysis of interinstitutional observer agreement in interpretation of dobutamine stress echocardiograms. J. Am. Coll. Cardiol. 27, 330-336 (1996).

6. Alagarsamy, S. et al. Comparison of clinical criteria with echocardiographic findings in diagnosing PDA in preterm infants. J. Perinat. Med. 33, 161-164 (2005).

7. de Boode, W. P. Clinical monitoring of systemic hemodynamics in critically ill newborns. Early Hum. Dev. 86, 137-141 (2010).

8. Bijnens, B. H., Cikes, M., Claus, P. \& Sutherland, G. R. Velocity and deformation imaging for the assessment of myocardial dysfunction. Eur. J. Echocardiogr. 10, 216-226 (2009).

9. Breatnach, C. R., Levy, P. T., James, A. T., Franklin, O. \& El-Khuffash, A. Novel echocardiography methods in the functional assessment of the newborn heart. Neonatology 110, 248-260 (2016).

10. Jain, A. et al. A comprehensive echocardiographic protocol for assessing neonatal right ventricular dimensions and function in the transitional period: normative data and z scores. J. Am. Soc. Echocardiogr. 27, 1293-1304 (2014).

11. Levy, P. T. et al. Maturational patterns of systolic ventricular deformation mechanics by two-dimensional speckle-tracking echocardiography in preterm infants over the first year of age. J. Am. Soc. Echocardiogr. 30, 685-698 (2017).

12. James, A., Corcoran, J. D., Mertens, L., Franklin, O. \& El-Khuffash, A. Left ventricular rotational mechanics in preterm infants less than 29 weeks' gestation over the first week after birth. J. Am. Soc. Echocardiogr. 28, 808-817 (2015).

13. Tee, M., Noble, J. A. \& Bluemke, D. A. Imaging techniques for cardiac strain and deformation: comparison of echocardiography, cardiac magnetic resonance and cardiac computed tomography. Expert Rev. Cardiovasc. Ther. 11, 221-231 (2013).

14. Geyer, H. et al. Assessment of myocardial mechanics using speckle tracking echocardiography: fundamentals and clinical applications. J. Am. Soc. Echocardiogr. 23, 351-369 (2010). quiz453-5.

15. Blessberger, H. \& Binder, T. Two dimensional speckle tracking echocardiography: clinical applications. Heart 96, 2032-2040 (2010).

16. Greenbaum, R. A., Ho, S. Y., Gibson, D. G., Becker, A. E. \& Anderson, R. H. Left ventricular fibre architecture in man. Br. Heart J. 45, 248-263 (1981).

17. Petitjean, C., Rougon, N. \& Cluzel, P. Assessment of myocardial function: a review of quantification methods and results using tagged MRI. J. Cardiovasc. Magn. Reson. 7, 501-516 (2005).
18. Levy, P. T. et al. Normal ranges of right ventricular systolic and diastolic strain measures in children: a systematic review and meta-analysis. J. Am. Soc. Echocardiogr. 27, 549-560 (2014).

19. de Waal, K., Phad, N., Lakkundi, A. \& Tan, P. Cardiac function after the immediate transitional period in very preterm infants using speckle tracking analysis. Pediatr. Cardiol. 37, 295-303 (2016).

20. Lorch, S. M., Ludomirsky, A. \& Singh, G. K. Maturational and growth-related changes in left ventricular longitudinal strain and strain rate measured by twodimensional speckle tracking echocardiography in healthy pediatric population. J. Am. Soc. Echocardiogr. 21, 1207-1215 (2008).

21. Voigt, J. U. et al. Definitions for a common standard for 2D speckle tracking echocardiography: consensus document of the EACVI/ASE/Industry Task Force to standardize deformation imaging. J. Am. Soc. Echocardiogr. 28, 183-193 (2015).

22. Boettler, P. et al. Heart rate effects on strain and strain rate in healthy children. $J$. Am. Soc. Echocardiogr. 18, 1121-1130 (2005).

23. Greenberg, N. L. et al. Doppler-derived myocardial systolic strain rate is a strong index of left ventricular contractility. Circulation 105, 99-105 (2002).

24. Weidemann, F. et al. Myocardial function defined by strain rate and strain during alterations in inotropic states and heart rate. Am. J. Physiol. Heart Circ. Physiol. 283, H792-H799 (2002).

25. Ferferieva, V. et al. The relative value of strain and strain rate for defining intrinsic myocardial function. Am. J. Physiol. Heart Circ. Physiol. 302, H188-H195 (2012).

26. James, A. T. et al. Longitudinal assessment of left and right myocardial function in preterm infants using strain and strain rate imaging. Neonatology 109, 69-75 (2016).

27. James, A. T., Corcoran, J. D., Hayes, B., Franklin, O. \& El-Khuffash, A. The effect of antenatal magnesium sulfate on left ventricular afterload and myocardial function measured using deformation and rotational mechanics imaging. J. Perinatol. 35, 913-918 (2015).

28. Kaul, S. et al. A suggested roadmap for cardiovascular ultrasound research for the future. J. Am. Soc. Echocardiogr. 24, 455-464 (2011).

29. Marwick, T. H. Will standardization make strain a standard measurement? J. Am. Soc. Echocardiogr. 25, 1204-1206 (2012).

30. Farsalinos, K. E. et al. Head-to-head comparison of global longitudinal strain measurements among nine different vendors: the EACVI/ASE inter-vendor comparison study. J. Am. Soc. Echocardiogr. 28, 1171-1181 (2015).

31. Gorcsan, J. 3rd \& Tanaka, H. Echocardiographic assessment of myocardial strain. J. Am. Coll. Cardiol. 58, 1401-1413 (2011).

32. de, W. K., Lakkundi, A. \& Othman, F. Speckle tracking echocardiography in very preterm infants: feasibility and reference values. Early Hum. Dev. 90, 275-279 (2014).

33. Pena, J. L. et al. Sequential changes of longitudinal and radial myocardial deformation indices in the healthy neonate heart. J. Am. Soc. Echocardiogr. 2, 294-300 (2010)

34. Helfer, S., Schmitz, L., Buhrer, C. \& Czernik, C. Tissue doppler-derived strain and strain rate during the first 28 days of life in very low birth weight infants. Echocardiography 31, 765-772 (2013).

35. Nestaas, E., Stoylen, A. \& Fugelseth, D. Myocardial performance assessment in neonates by one-segment strain and strain rate analysis by tissue Doppler - a quality improvement cohort study. BMJ Open. 2, e001636 (2012).

36. Nestaas, E., Stoylen, A., Sandvik, L., Brunvand, L. \& Fugelseth, D. Feasibility and reliability of strain and strain rate measurement in neonates by optimizing the analysis parameters settings. Ultrasound Med. Biol. 33, 270-278 (2007).

37. Nestaas, E., Stoylen, A., Brunvand, L. \& Fugelseth, D. Tissue Doppler derived longitudinal strain and strain rate during the first 3 days of life in healthy term neonates. Pediatr. Res. 65, 357-362 (2009).

38. Poon, C. Y., Edwards, J. M., Joshi, S., Kotecha, S. \& Fraser, A. G. Optimization of myocardial deformation imaging in term and preterm infants. Eur. J. Echocardiogr. 12, 247-254 (2011).

39. Helfer, S., Schmitz, L., Buhrer, C. \& Czernik, C. Reproducibility and optimization of analysis parameters of tissue doppler-derived strain and strain rate measurements for very low birth weight infants. Echocardiography 30, 1219-1226 (2013).

40. Joshi, S. et al. Reproducibility of myocardial velocity and deformation imaging in term and preterm infants. Eur. J. Echocardiogr. 11, 44-50 (2010).

41. James, A. T. et al. Assessment of myocardial performance in preterm infants less than 29 weeks gestation during the transitional period. Early Hum. Dev. 90, 829-835 (2014).

42. Teske, A. J. et al. Echocardiographic quantification of myocardial function using tissue deformation imaging, a guide to image acquisition and analysis using tissue Doppler and speckle tracking. Cardiovasc. Ultrasound 5, 27 (2007).

43. Nestaas, E., Stoylen, A. \& Fugelseth, D. Optimal types of probe, and tissue Doppler frame rates, for use during tissue Doppler recording and off-line analysis of strain and strain rate in neonates at term. Cardiol. Young 18, 502-511 (2008). 
44. Aase, S. A., Stoylen, A., Ingul, C. B., Frigstad, S. \& Torp, H. Automatic timing of aortic valve closure in apical tissue Doppler images. Ultrasound Med. Biol. 32, 19-27 (2006)

45. Pena, J. L. et al. Quantification of regional left and right ventricular deformation indices in healthy neonates by using strain rate and strain imaging. J. Am. Soc. Echocardiogr. 22, 369-375 (2009).

46. Nestaas, E., Skranes, J. H., Stoylen, A., Brunvand, L. \& Fugelseth, D. The myocardial function during and after whole-body therapeutic hypothermia for hypoxic-ischemic encephalopathy, a cohort study. Early Hum. Dev. 90, 247-252 (2014).

47. James, A. T., Corcoran, J. D., McNamara, P. J., Franklin, O. \& El-Khuffash, A. F. The effect of milrinone on right and left ventricular function when used as a rescue therapy for term infants with pulmonary hypertension. Cardiol. Young 26, 90-99 (2016).

48. Koopman, L. P. et al. Comparison between different speckle tracking and color tissue doppler techniques to measure global and regional myocardial deformation in children. J. Am. Soc. Echocardiogr. 23, 919-928 (2010).

49. Khan, U., Hjertaas, J. J., Greve, G. \& Matre, K. Optimal acquisition settings for speckle tracking echocardiography-derived strains in infants: an in vitro study. Ultrasound Med. Biol. 42, 1660-1670 (2016).

50. Levy, P. T. et al. Reference ranges of left ventricular strain measures by twodimensional speckle-tracking echocardiography in children: a systematic review and meta-analysis. J. Am. Soc. Echocardiogr. 29, 209-225 (2016).

51. Jain, A. et al. Left ventricular function in healthy term neonates during the transitional period. J. Pediatr. 182, 197-203 (2017).

52. Rudski, L. G. et al. Guidelines for the echocardiographic assessment of the right heart in adults: a report from the American Society of Echocardiography endorsed by the European Association of Echocardiography, a registered branch of the European Society of Cardiology, and the Canadian Society of Echocardiography. J. Am. Soc. Echocardiogr. 23, 685-713 (2010).

53. Nasu, Y. et al. Longitudinal systolic strain of the bilayered ventricular septum during the first $72 \mathrm{~h}$ of life in preterm infants. J. Echocardiogr. 13, 90-99 (2015).

54. Sanchez, A. A. et al. Effects of frame rate on two-dimensional speckle trackingderived measurements of myocardial deformation in premature infants. Echocardiography 32, 839-847 (2015).

55. Maskatia, S. A. et al. Longitudinal changes and interobserver variability of systolic myocardial deformation values in a prospective cohort of healthy fetuses across gestation and after delivery. J. Am. Soc. Echocardiogr. 29, 341-349 (2016).

56. Marcus, K. A. et al. Reference values for myocardial two-dimensional strain echocardiography in a healthy pediatric and young adult cohort. J. Am. Soc. Echocardiogr. 24, 625-636 (2011).

57. Klitsie, L. M., Roest, A. A., Haak, M. C., Blom, N. A. \& Ten Harkel, A. D. Longitudinal follow-up of ventricular performance in healthy neonates. Early Hum. Dev. 89, 993-997 (2013).

58. Schubert, U., Muller, M., Norman, M. \& Abdul-Khaliq, H. Transition from fetal to neonatal life: changes in cardiac function assessed by speckle-tracking echocardiography. Early Hum. Dev. 89, 803-808 (2013).

59. Schubert, U., Muller, M., Abdul-Khaliq, H. \& Norman, M. Preterm birth is associated with altered myocardial function in infancy. J. Am. Soc. Echocardiogr. 29, 670-678 (2016).

60. De Waal, K., Phad, N., Lakkundi, A. \& Tan, P. Post-transitional adaptation of the left heart in uncomplicated, very preterm infants. Cardiol. Young. 2017 Aug; 27:11671173.

61. Haque, U. et al. Right ventricular performance using myocardial deformation imaging in infants with bronchopulmonary dysplasia. J. Perinatol. 37, 81-87 (2017).

62. Sehgal, A., Doctor, T. \& Menahem, S. Cardiac function and arterial indices in infants born small for gestational age: analysis by speckle tracking. Acta Paediatr. 103, e49-e54 (2014).

63. Al-Biltagi, M., Tolba, O. A., Rowisha, M. A., Mahfouz, A. \& Elewa, M. A. Speckle tracking and myocardial tissue imaging in infant of diabetic mother with gestational and pregestational diabetes. Pediatr. Cardiol. 36, 445-453 (2015).
64. Hirose, A. et al. Evolution of left ventricular function in the preterm infant. J. Am. Soc. Echocardiogr. 28, 302-308 (2015).

65. Czernik, C. et al. Development of left ventricular longitudinal speckle tracking echocardiography in very low birth weight infants with and without bronchopulmonary dysplasia during the neonatal period. PLOS ONE 9, e106504 (2014).

66. Sehgal, A., Wong, F. \& Menahem, S. Speckle tracking derived strain in infants with severe perinatal asphyxia: a comparative case control study. Cardiovasc. Ultrasound 11, 34 (2013).

67. Levy, P. T. \& El-Khuffash, A. Pulmonary arterial hypertension after ibuprofen treatment in the first week of life? J. Pediatr. 182, 408-409 (2017).

68. Liao, W. Q., Zhou, H. Y., Chen, G. C., Zou, M. \& Lv, X. [Left ventricular function in newborn infants of mothers with gestational diabetes mellitus]. Zhongguo Dang Dai Er Ke Za Zhi. 14, 575-577 (2012).

69. Sengupta, P. P., Tajik, A. J., Chandrasekaran, K. \& Khandheria, B. K. Twist mechanics of the left ventricle: principles and application. JACC CardiovasC. Imaging 1, 366-376 (2008).

70. Buckberg, G. et al. Ventricular torsion and untwisting: further insights into mechanics and timing interdependence: a viewpoint. Echocardiography 28, 782-804 (2011).

71. Burns, A. T., La, G. A., Prior, D. L. \& Macisaac, A. I. Left ventricular torsion parameters are affected by acute changes in load. Echocardiography 27, 407-414 (2010).

72. Ramani, G. V., Bazaz, R., Edelman, K. \& Lopez-Candales, A. Pulmonary hypertension affects left ventricular basal twist: a novel use for speckle-tracking imaging. Echocardiography 26, 44-51 (2009).

73. Zhang, Y., Zhou, Q. C., Pu, D. R., Zou, L. \& Tan, Y. Differences in left ventricular twist related to age: speckle tracking echocardiographic data for healthy volunteers from neonate to age 70 years. Echocardiography 27, 1205-1210 (2010).

74. Kim, H. J. et al. Normal left ventricular torsion mechanics in healthy children: age related changes of torsion parameters are closely related to changes in heart rate. Korean Circ. J. 45, 131-140 (2015).

75. Al-Naami, G. H. Torsion of young hearts: a speckle tracking study of normal infants, children, and adolescents. Eur. J. Echocardiogr. 11, 853-862 (2010).

76. Notomi, Y. et al. Measurement of ventricular torsion by two-dimensional ultrasound speckle tracking imaging. J. Am. Coll. Cardiol. 45, 2034-2041 (2005).

77. Xie, M. et al. Left ventricular torsion abnormalities in patients after the arterial switch operation for transposition of the great arteries with intact ventricular septum. Int. J. Cardiol. 168, 4631-4637 (2013).

78. Breatnach, C. R. et al. Left ventricular rotational mechanics in infants with hypoxic ischemic encephalopathy and preterm infants at 36 weeks postmenstrual age: a comparison with healthy term controls. Echocardiography 34, 232-239 (2017).

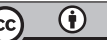

Open Access This article is licensed under a Creative Commons Attribution 4.0 International License, which permits use, sharing adaptation, distribution and reproduction in any medium or format, as long as you give appropriate credit to the original author(s) and the source, provide a link to the Creative Commons license, and indicate if changes were made. The images or other third party material in this article are included in the article's Creative Commons license, unless indicated otherwise in a credit line to the material. If material is not included in the article's Creative Commons license and your intended use is not permitted by statutory regulation or exceeds the permitted use, you will need to obtain permission directly from the copyright holder. To view a copy of this license, visit http://creativecommons. org/licenses/by/4.0/.

(c) The Author(s) 2018 